QUARTERLY OF APPLIED MATHEMATICS

VOLUME LXIII, NUMBER 3

SEPTEMBER 2005, PAGES 401-427

S 0033-569X(05)00961-8

Article electronically published on August 17, 2005

\title{
DELTA-SHOCK WAVE TYPE SOLUTION OF HYPERBOLIC SYSTEMS OF CONSERVATION LAWS
}

\author{
BY \\ V. G. DANILOV (Department of Mathematics, Moscow Technical University of Communication and \\ Informatics, Aviamotornaya, 8a, 111024, Moscow, Russia) \\ AND \\ V. M. SHELKOVICH (Department of Mathematics, St.-Petersburg State Architecture and Civil \\ Engineering University, 2 Krasnoarmeiskaya 4, 190005, St. Petersburg, Russia)
}

Abstract. For two classes of hyperbolic systems of conservation laws new definitions of a $\delta$-shock wave type solution are introduced. These two definitions give natural generalizations of the classical definition of the weak solutions. It is relevant to the notion of $\delta$-shocks. The weak asymptotics method developed by the authors is used to describe the propagation of $\delta$-shock waves to the three types of systems of conservation laws and derive the corresponding Rankine-Hugoniot conditions for $\delta$-shocks.

\section{Introduction.}

1.1. Singular solutions to hyperbolic systems of conservation laws. One of the approaches to solving the problems related to singular solutions of quasilinear equations was developed by the authors in [3]-8] (see also [2], 23]). In these papers, the authors developed a new asymptotics method - the weak asymptotics method - 'for studying the dynamics of propagation and interaction of different singularities of quasilinear differential equations and hyperbolic systems of conservation laws (infinitely narrow $\delta$-solitons, shocks, $\delta$-shocks).

In this paper in the framework of the weak asymptotics method for two classes of hyperbolic systems of conservation laws we introduce a new definition of a $\delta$-shock wave

Received August 5, 2003.

2000 Mathematics Subject Classification. Primary 35L65; Secondary 35L67, 76L05.

Key words and phrases. Hyperbolic systems of conservation laws, zero-pressure gas dynamics system, delta-shock wave type solutions, the Rankine-Hugoniot conditions of delta-shocks, the weak asymptotics method.

The first author (V. D.) was supported in part by Grant 05-01-00912 of Russian Foundation for Basic Research, SEP-CONACYT Grant 41421, SEP-CONACYT Grant 43208 (Mexico).

The second author (V. S.) was supported in part by DFG Project 436 RUS 113/593/3, Grant 02-0100483 of Russian Foundation for Basic Research, and SEP-CONACYT Grant 41421, SEP-CONACYT Grant 43208 (Mexico).

E-mail address: danilov@miem.edu.ru

E-mail address: shelkv@vs1567.spb.edu 
type solution by integral identities. This definition gives natural generalizations of the classical definition of the weak $L^{\infty}$-solutions and specifies the definition of measuresolutions given in [1, 26, 28.

By using the weak asymptotics method we describe the propagation of $\delta$-shock waves to the three types of systems of conservation laws and derive the corresponding RankineHugoniot conditions for $\delta$-shocks. Among these systems are the well-known KeyfitzKranzer system (1.7) and zero-pressure gas dynamics system (1.9).

For all these systems we construct weak asymptotic solutions (approximating solutions in the weak sense) and prove that the weak limits of these weak asymptotic solutions satisfy our definition.

Consider the following hyperbolic systems of conservation laws

$$
\begin{aligned}
& L_{1}[u, v]=u_{t}+(F(u, v))_{x}=0 \\
& L_{2}[u, v]=v_{t}+(G(u, v))_{x}=0,
\end{aligned}
$$

and

$$
\begin{aligned}
& L_{1}[u, v]=v_{t}+(G(u, v))_{x}=0, \\
& L_{2}[u, v]=(u v)_{t}+(H(u, v))_{x}=0,
\end{aligned}
$$

where $F(u, v), G(u, v), H(u, v)$ are smooth functions, linear with respect to $v ; u=$ $u(x, t), v=v(x, t) \in \mathbb{R} ;$ and $x \in \mathbb{R}$.

As is well known, hyperbolic systems of conservation laws, even in the case of smooth (and, certainly, in the case of discontinuous) initial data $\left(u^{0}(x), v^{0}(x)\right)$, may have discontinuous solutions. In this case, it is said that a pair of functions $(u(x, t), v(x, t)) \in$ $L^{\infty}\left(\mathbb{R} \times(0, \infty) ; \mathbb{R}^{2}\right)$ is a generalized solution of the Cauchy problem (1.1) with the initial data $\left(u^{0}(x), v^{0}(x)\right)$ if the integral identities

$$
\begin{aligned}
& \int_{0}^{\infty} \int\left(u \varphi_{t}+F(u, v) \varphi_{x}\right) d x d t+\int u^{0}(x) \varphi(x, 0) d x=0, \\
& \int_{0}^{\infty} \int\left(v \varphi_{t}+G(u, v) \varphi_{x}\right) d x d t+\int v^{0}(x) \varphi(x, 0) d x=0
\end{aligned}
$$

hold for all compactly supported test functions $\varphi(x, t) \in \mathcal{D}(\mathbb{R} \times[0, \infty))$, where $\int \cdot d x$ denotes an improper integral $\int_{-\infty}^{\infty} \cdot d x$. A definition of a generalized solution of system (1.2) can be introduced in the same way as for system (1.1).

Consider the Cauchy problem for system (1.1) or (1.2) with initial data

$$
u^{0}(x)=u_{0}+u_{1} H(-x), \quad v^{0}(x)=v_{0}+v_{1} H(-x),
$$

where $u_{0}, u_{1}, v_{0}$, and $v_{1}$ are constants and $H(\xi)$ is the Heaviside function. Here as was already mentioned above, the functions $F(u, v), G(u, v), H(u, v)$ are linear with respect to $v$. As was shown in [1, 6]-[15, 26, 28, in order to solve this problem for some "nonclassical cases", it is necessary to introduce new elementary singularities called $\delta$ shock waves. These are generalized solutions of hyperbolic systems of conservation laws of the form

$$
\begin{aligned}
& u(x, t)=u_{0}+u_{1} H(-x+c t), \\
& v(x, t)=v_{0}+v_{1} H(-x+c t)+e(t) \delta(-x+c t),
\end{aligned}
$$

where $e(t)$ is a smooth function such that $e(0)=0$ and $\delta(\xi)$ is the Dirac delta function. 
At present several approaches to constructing such solutions are known. The actual difficulty in defining such solutions arises due to the fact that (as follows from (1.1), (1.2), (1.5)), to introduce a definition of the $\delta$-shock wave type solution, one needs to define singular superpositions of distributions (for example, the product of the Heaviside function and the $\delta$-function). We also need to define in which sense a distributional solution (for example, (1.5)) satisfies a nonlinear system.

In [13], a $\delta$-shock wave type solution of the system

$$
u_{t}+\left(\frac{1}{2} u^{2}\right)_{x}=0, \quad v_{t}+(u v)_{x}=0,
$$

(here $F(u, v)=u^{2} / 2, G(u, v)=v u$ ) with the initial data (1.4), is defined as a weak limit of the solution $(u(x, t, \varepsilon), v(x, t, \varepsilon))$ of the parabolic regularization

$$
u_{t}+\left(\frac{1}{2} u^{2}\right)_{x}=\varepsilon u_{x x}, \quad v_{t}+(u v)_{x}=\varepsilon v_{x x},
$$

as $\varepsilon \rightarrow+0$. In [12], to obtain a $\delta$-shock wave type solution of the system

$$
\begin{gathered}
L_{11}[u, v]=u_{t}+(f(u))_{x}=0, \\
L_{12}[u, v]=v_{t}+(g(u) v)_{x}=0,
\end{gathered}
$$

(here $F(u, v)=f(u), G(u, v)=v g(u)$ ), this system is reduced to a system of HamiltonJacobi equations, and then the Lax formula is used. In 15, to construct a $\delta$-shock wave type solution of system (1.8) for the case $g(u)=f^{\prime}(u)$, the problem of multiplication of distributions is solved by using the definition of Volpert's averaged superposition [27. In [19, the nonconservative product of singular functions is defined by generalization of Volpert's ideas.

In 14, in order to construct an approximate $\delta$-shock type solution of the system

$$
\begin{aligned}
& L_{21}[u, v]=u_{t}+\left(u^{2}-v\right)_{x}=0, \\
& L_{22}[u, v]=v_{t}+\left(\frac{1}{3} u^{3}-u\right)_{x}=0,
\end{aligned}
$$

(here $F(u, v)=u^{2}-v, G(u, v)=u^{3}-u$ ), the Colombeau theory approach, as well as the Dafermos-DiPerna regularization (under the assumption that Dafermos profiles exist), and the box approximations are used. However, the notion of a generalized solution has not been defined. It is not clear in what sense $\delta$-shock solution (1.5) satisfies system (1.7). Using the definition of a $\delta$-shock wave type solution [7, 8], the exact $\delta$-shock wave type solutions for system (1.7) with piecewise constant initial data was first constructed in 24]. (see Corollary 3.3).

In [20] in the framework of the Colombeau theory approach, approximate $\delta$-shock type solutions were constructed for some classes of systems.

In [26] for the system

$$
u_{t}+\left(u^{2}\right)_{x}=0, \quad v_{t}+(u v)_{x}=0,
$$

(here $F(u, v)=u^{2}, G(u, v)=v u$ ), in [1], [16] for the system

$$
\begin{aligned}
& L_{31}[u, v]=v_{t}+(v u)_{x}=0, \\
& L_{32}[u, v]=(v u)_{t}+\left(v u^{2}\right)_{x}=0,
\end{aligned}
$$


(here $G(u, v)=u v, H(u, v)=u^{2} v ; v \geq 0$ is the density, $u$ is the velocity), and in 28] for the system

$$
v_{t}+(v f(u))_{x}=0, \quad(v u)_{t}+(v u f(u))_{x}=0
$$

(here $G(u, v)=v f(u), H(u, v)=v u f(u))$ with the initial data (1.4), the $\delta$-shock wave type solution is defined as a measure-valued solution (see also [25]).

Recall the definition of a measure-valued solution. Let $B M(\mathbb{R})$ be the space of bounded Borel measures and let $v(x, t) \in C(B M(\mathbb{R}),[0, \infty))$ and $u(x, t) \in L^{\infty}\left(L^{\infty}(\mathbb{R}),[0, \infty)\right)$. A pair $(u, v)$ is said to be a measure-valued solution of the Cauchy problem (1.10), (1.4) if

$$
\begin{aligned}
\int_{0}^{\infty} \int\left(\varphi_{t}+f(u) \varphi_{x}\right) v(d x, t) & =0 \\
\int_{0}^{\infty} \int u\left(\varphi_{t}+f(u) \varphi_{x}\right) v(d x, t) & =0
\end{aligned}
$$

for all $\varphi(x, t) \in \mathcal{D}(\mathbb{R} \times[0, \infty))$.

Within the framework of this definition in [26] for system (1.8), in [1] for system (1.9), and in 28 for system (1.10), the following formulas for $\delta$-shock waves derived

$$
(u(x, t), v(x, t))=\left\{\begin{aligned}
\left(u^{-}, v^{-}\right), & x<\phi(t), \\
\left(u_{\delta}, w(t) \delta(x-\phi(t))\right), & x=\phi(t), \\
\left(u^{+}, v^{+}\right), & x>\phi(t) .
\end{aligned}\right.
$$

Here $u^{-}, u^{+}$and $u_{\delta}$ are the velocities before the discontinuity, after the discontinuity, and at the point of discontinuity, respectively, and $\phi(t)=\sigma_{\delta} t$ is the equation for the discontinuity line.

In [9], the global $\delta$-shock wave type solution was obtained for system (1.9). By using the Colombeau theory approach, in [11, an approximating solution of the Cauchy problem is constructed for this system.

The study of systems, which admit $\delta$-shock wave type solutions is very important in applications, because systems of this type often arise in modelling physical processes in gas dynamics, magnetohydrodynamics, filtration theory. In particular, there are large numbers of papers where "zero-pressure gas dynamics system" (1.9) is studied. In the multidimensional case this system was used to describe the formation of large-scale structures of the universe [22, 29]. This system is also used to describe the motion of free particles which stick under collision.

1.2. Main results. Below, in Subsec. 1.3. for two classes of hyperbolic systems of conservation laws we present the Definition 1.1 and the Definition 1.2 of $\delta$-shock wave type solutions. These definitions are natural generalizations of the classical definition (1.3) for $L^{\infty}$ solutions and specify the measure-solutions definition (1.11) (see [1], 26], 28]) for the case in which the support of the singular part of the measure $v$ is a union of pieces of smooth curves. According to our Definitions 1.1, 1.2 a generalized $\delta$-shock wave type solution is a pair of distributions $(u(x, t), v(x, t))$ unlike the definition (1.11), where $v(d x, t)$ is a measure and $u(x, t)$ is understood as a measurable function defined $v(d x, t)$ a.e.

In Subsec. 1.4 we introduce the notion of a weak asymptotic solution of the Cauchy problem, which is one of the most important notions in the weak asymptotics method. 
We apply the weak asymptotics method in order to solve the Cauchy problem for system (1.6) (in Sec. 22), and for Keyfitz-Kranzer system (1.7) (in Sec. 3), with a special form of the initial data

$$
\begin{aligned}
& u^{0}(x)=u_{0}^{0}(x)+u_{1}^{0}(x) H(-x), \\
& v^{0}(x)=v_{0}^{0}(x)+v_{1}^{0}(x) H(-x)+e^{0} \delta(-x),
\end{aligned}
$$

where $u_{k}^{0}(\mathrm{x}), v_{k}^{0}(x), \quad k=0,1$ are given smooth functions, $e^{0}$ is a given constant. Here the initial data (1.13) can contain a $\delta$-function, while in most papers on $\delta$-shocks, initial data without a $\delta$-function are considered, because the technical base of these papers is connected with self-similar solutions.

The $\delta$-shock wave type solutions of the Cauchy problems (1.6), (1.13) and (1.7), (1.13) given by Theorem 2.4. Corollary 2.5 and Theorem 3.2, Corollary 3.3, respectively. The fifth and sixth equations of systems (2.8), (3.15) are the Rankine-Hugoniot conditions of $\delta$-shocks. Here the fifth equation of systems (2.8), (3.15) is the "standard" RankineHugoniot condition. The right-hand side of the sixth equation of systems (2.8), (3.15) called the Rankine-Hugoniot deficit.

If $e^{0}=0$, and the initial data are piecewise constant, our results on a weak asymptotic solution of Keyfitz-Kranzer system (1.7) (constructed by Theorem 3.1) coincide with the main statements on an approximate solution from [14]. Namely, in [14 a particular case of an approximate solution (1.20), (3.2) of the Cauchy problem (1.7), (1.13) with piecewise constant initial data was constructed (see Remark 3.4).

The Cauchy problem and the Rankine-Hugoniot conditions for system (1.9) are essentially different from the Cauchy problem and the Rankine-Hugoniot conditions for system (1.1). As will be shown in Sec.4, in contrast to the Cauchy problems (1.6), (1.13) and (1.7), (1.13), for the Cauchy problem (1.9), (1.13) the system of ordinary differential equations (two last Eq. (4.9) ) that determines the trajectory of a singularity and the coefficient of the $\delta$-function, i.e., the Rankine-Hugoniot conditions of $\delta$-shocks, is a system of second-order equations. Thus, in the general case, for the unique solvability of the Cauchy problem posed for this system, it is necessary to specify the initial velocity along the trajectory of $\delta$-shock wave. We study this in detail at the end of Sec. 4. Here we only note that the value of the initial velocity is not determined by the initial data (1.13). Hence the Cauchy problem (1.9), (1.13) has a family of solutions parameterized by the parameter of the initial velocity.

Thus, in Sec.4, to solve the Cauchy problem for system (1.9), in the initial data (1.13) we introduce the initial velocity of singularity, and instead of the initial data (1.13), we use the initial data

$$
\begin{aligned}
u^{0}(x) & =u_{0}^{0}(x)+u_{1}^{0}(x) H(-x), \\
v^{0}(x) & =v_{0}^{0}(x)+v_{1}^{0}(x) H(-x)+e^{0} \delta(-x) . \\
\dot{\phi}(0) & =\phi^{1}
\end{aligned}
$$

where $u_{k}^{0}(\mathrm{x}), v_{k}^{0}(x), \quad k=0,1$ are given smooth functions, $e^{0}, \phi^{1}$ are given constants, $u_{1}^{0}(0)>0$, and $\cdot=\frac{d}{d t}$.

The $\delta$-shock wave type solutions of the Cauchy problem (1.9), (1.14) is given by Theorem 4.2. The case of the piecewise constant initial data (1.14) is given by Theorem 4.3 
and Corollary 4.4. The right-hand side of the fifth and sixth equation of systems (4.9) are the Rankine-Hugoniot deficits.

Formulas for the trajectory of a singularity $\phi(t)$ and for the coefficient $e(t)$ of the $\delta$ function in Theorem 4.3 and Corollary 4.4 coincide with the analogous formulas from [1], [16, 25] if we identify the velocity on the discontinuity line $x=\phi(t)$ in formula (1.12) with the phase velocity of nonlinear wave:

$$
u_{\delta}=\dot{\phi}(t)
$$

As mentioned in Subsec. 1.1, the problem of defining $\delta$-shock wave type solution of the Cauchy problem is connected with the construction of singular superpositions (products) of distributions. In this paper, in the framework of the weak asymptotics method by relations (2.9), (3.16), (3.17), (4.10), (4.11), in fact, we define the singular superpositions of distributions. In the background of these formulas there is the construction of asymptotic subalgebras of distributions, but in the description of our technique we omit the algebraic aspects which are given in detail in [2, 3], 23.

In conclusion, we note that the weak asymptotic method allows one to study the initial conditions having the form of a linear combination of functions contained in (1.13) or (1.14) and hence to solve the problem of $\delta$-shock waves interaction. For the case of system (1.6) this problem was solved in [6]-8].

1.3. $\delta$-Shock wave type solution. In what follows, we introduce definitions of a $\delta$-shock wave type solution for systems (1.1) and (1.9). Recall that the functions $F(u, v), G(u, v)$ are linear with respect to $v$.

Suppose that $\Gamma=\left\{\gamma_{i}: i \in I\right\}$ is a graph in the upper half-plane $\{(x, t): x \in \mathbb{R}, t \in$ $[0, \infty)\} \in \mathbb{R}^{2}$ containing smooth $\operatorname{arcs} \gamma_{i}, i \in I$, and $I$ is a finite set. By $I_{0}$ we denote a subset of $I$ such that an arc $\gamma_{k}$ for $k \in I_{0}$ starting from the points of the $x$-axis. Denote by $\Gamma_{0}=\left\{x_{k}^{0}: k \in I_{0}\right\}$ the set of initial points of $\operatorname{arcs} \gamma_{k}, k \in I_{0}$.

Consider $\delta$-shock wave type initial data $\left(u^{0}(x), v^{0}(x)\right)$, where

$$
v^{0}(x)=V^{0}(x)+e^{0} \delta\left(\Gamma_{0}\right),
$$

$u^{0}, V^{0} \in L^{\infty}(\mathbb{R} ; \mathbb{R}), \quad e^{0} \delta\left(\Gamma_{0}\right)=\sum_{k \in I_{0}} e_{k}^{0} \delta\left(x-x_{k}^{0}\right), \quad e_{k}^{0}$ are constants, $k \in I_{0}$.

Definition 1.1. A pair of distributions $(u(x, t), v(x, t))$ and graph $\Gamma$, where $v(x, t)$ is represented in form of the sum

$$
v(x, t)=V(x, t)+e(x, t) \delta(\Gamma),
$$

$u, V \in L^{\infty}(\mathbb{R} \times(0, \infty) ; \mathbb{R}), \quad e(x, t) \delta(\Gamma)=\sum_{i \in I} e_{i}(x, t) \delta\left(\gamma_{i}\right), \quad e_{i}(x, t) \in C(\Gamma), i \in I$, is called a generalized $\delta$-shock wave type solution of system (1.1) with the $\delta$-shock wave type initial data $\left(u^{0}(x), v^{0}(x)\right)$ if the integral identities

$$
\begin{aligned}
\int_{0}^{\infty} \int\left(u \varphi_{t}+F(u, V) \varphi_{x}\right) d x d t+\int u^{0}(x) \varphi(x, 0) d x & =0 \\
\int_{0}^{\infty} \int\left(V \varphi_{t}+G(u, V) \varphi_{x}\right) d x d t & \\
+\sum_{i \in I} \int_{\gamma_{i}} e_{i}(x, t) \frac{\partial \varphi(x, t)}{\partial \mathbf{l}} d l & \\
+\int V^{0}(x) \varphi(x, 0) d x+\sum_{k \in I_{0}} e_{k}^{0} \varphi\left(x_{k}^{0}, 0\right) & =0
\end{aligned}
$$


hold for all test functions $\varphi(x, t) \in \mathcal{D}(\mathbb{R} \times[0, \infty))$, where $\frac{\partial \varphi(x, t)}{\partial \mathbf{l}}$ is the tangential derivative on the graph $\Gamma, \int_{\gamma_{i}} \cdot d l$ is a line integral over the $\operatorname{arc} \gamma_{i}$.

For instance, the graph $\Gamma$ containing only one arc $\{(x, t): x=c t\}, \phi(0)=0$ corresponds to solution (1.5).

Now we introduce definitions of a $\delta$-shock wave type solution for systems (1.9). Suppose that arcs of the graph $\Gamma=\left\{\gamma_{i}: i \in I\right\}$ have the form $\gamma_{i}=\left\{(x, t): x=\phi_{i}(t)\right\}, i \in I$.

Definition 1.2. A pair of distributions $(u(x, t), v(x, t))$ and graph $\Gamma$ from Definition 1.1 is called a generalized $\delta$-shock wave type solution of system (1.9) with the $\delta$-shock wave type initial data $\left(u^{0}(x), v^{0}(x) ; \dot{\phi}_{i}(0), i \in I_{0}\right)$ if the integral identities

$$
\begin{aligned}
\int_{0}^{\infty} \int\left(V \varphi_{t}+u V \varphi_{x}\right) d x d t & \\
+\sum_{i \in I} \int_{\gamma_{i}} e_{i}(x, t) \frac{\partial \varphi(x, t)}{\partial \mathbf{l}} d l & \\
+\int V^{0}(x) \varphi(x, 0) d x+\sum_{k \in I_{0}} e_{k}^{0} \varphi\left(x_{k}^{0}, 0\right) & =0 \\
\int_{0}^{\infty} \int\left(u V \varphi_{t}+u^{2} V \varphi_{x}\right) d x d t & \\
+\sum_{i \in I} \int_{\gamma_{i}} e_{i}(x, t) \dot{\phi}_{i}(t) \frac{\partial \varphi(x, t)}{\partial \mathbf{l}} d l & \\
+\int u^{0}(x) V^{0}(x) \varphi(x, 0) d x+\sum_{k \in I_{0}} e_{k}^{0} \dot{\phi}_{k}(0) \varphi\left(x_{k}^{0}, 0\right) & =0
\end{aligned}
$$

hold for all $\varphi(x, t) \in \mathcal{D}(\mathbb{R} \times[0, \infty))$.

According to Subsec. 1.2, in Definition 1.2 instead of the $\delta$-shock wave type initial data $\left(u^{0}(x), v^{0}(x)\right)$, we use the $\delta$-shock wave type initial data $\left(u^{0}(x), v^{0}(x) ; \dot{\phi}_{i}(0), i \in I_{0}\right)$, where $\dot{\phi}_{i}(0)$ is the initial velocity, $i \in I_{0}$.

Thus, as mentioned above, for system (1.9) in addition to the initial data (1.13) we add the initial velocity $\dot{\phi}(0)$ and use the initial data (1.14).

As in [10, 14], 26], to solve the Cauchy problems (1.1), (1.13) or (1.9), (1.14), we shall use the "overcompression" condition (for details, see [17]):

$$
\begin{aligned}
& \lambda_{1}\left(u_{+}, v_{+}\right) \leq \dot{\phi}(t) \leq \lambda_{1}\left(u_{-}, v_{-}\right), \\
& \lambda_{2}\left(u_{+}, v_{+}\right) \leq \dot{\phi}(t) \leq \lambda_{2}\left(u_{-}, v_{-}\right),
\end{aligned}
$$

as the admissibility condition for the $\delta$-shocks. Here $\lambda_{1}(u, v), \lambda_{2}(u, v)$ are the eigenvalues of the characteristic matrix of system (1.1) or (1.9), $\dot{\phi}(t)$ is the velocity of the motion of the $\delta$-shock wave, and $u_{-}, v_{-}$and $u_{+}, v_{+}$are the respective left- and right-hand values of $u, v$ on the discontinuity curve $x=\phi(t)$. Condition (1.17) means that all characteristic lines on both sides of the discontinuity are in-coming.

1.4. Weak asymptotic solution. Next, we introduce the definition of a weak asymptotic solution. We shall write $f(x, t, \varepsilon)=O_{\mathcal{D}^{\prime}}\left(\varepsilon^{\alpha}\right)$, if $f(x, t, \varepsilon) \in \mathcal{D}^{\prime}(\mathbb{R})$ is a distribution such that for any test function $\psi(x) \in \mathcal{D}\left(\mathbb{R}_{x}\right)$ we have

$$
\langle f(x, t, \varepsilon), \psi(x)\rangle=O\left(\varepsilon^{\alpha}\right),
$$

where $O\left(\varepsilon^{\alpha}\right)$ denotes a function continuous in $t$ that admits the usual estimate $\left|O\left(\varepsilon^{\alpha}\right)\right| \leq$ const $\varepsilon^{\alpha}$ uniform in $t$. The relation $o_{\mathcal{D}^{\prime}}\left(\varepsilon^{\alpha}\right)$ is understood in a corresponding way. 
Definition 1.3. A pair of functions $(u(x, t, \varepsilon), v(x, t, \varepsilon))$ smooth as $\varepsilon>0$ and graph $\Gamma$ is called a weak asymptotic solution of the Cauchy problem for system (1.1) with the $\delta$-shock wave type initial data $\left(u^{0}, v^{0}\right)$ if the relations 1

$$
\begin{aligned}
L_{1}[u(x, t, \varepsilon), v(x, t, \varepsilon)] & =O_{\mathcal{D}^{\prime}}(\varepsilon), \\
L_{2}[u(x, t, \varepsilon), v(x, t, \varepsilon)] & =O_{\mathcal{D}^{\prime}}(\varepsilon), \\
u(x, 0, \varepsilon) & =u^{0}(x)+O_{\mathcal{D}^{\prime}}(\varepsilon), \\
v(x, 0, \varepsilon) & =v^{0}(x)+O_{\mathcal{D}^{\prime}}(\varepsilon) .
\end{aligned}
$$

hold.

A definition of a weak asymptotic solution of the Cauchy problem for system (1.9) with the $\delta$-shock wave type initial data $\left(u^{0}(x), v^{0}(x) ; \dot{\phi}_{i}(0), i \in I_{0}\right)$ can be introduced in the same way.

Within the framework of the weak asymptotics method, we find a generalized $\delta$-shock wave type solution $(u(x, t), v(x, t))$ of the Cauchy problem as the weak limit (in the sense of the space of distributions $\left.\mathcal{D}^{\prime}(\mathbb{R} \times[0, \infty))\right)$

$$
\begin{aligned}
& u(x, t)=\lim _{\varepsilon \rightarrow+0} u(x, t, \varepsilon), \\
& v(x, t)=\lim _{\varepsilon \rightarrow+0} v(x, t, \varepsilon),
\end{aligned}
$$

where $(u(x, t, \varepsilon), v(x, t, \varepsilon))$ is a weak asymptotic solution of this problem.

Multiplying the first two relations in (1.18) by a test function $\varphi(x, t) \in \mathcal{D}(\mathbb{R} \times[0, \infty))$, integrating these relations by parts and then passing to the limit as $\varepsilon \rightarrow+0$, we shall prove that the pair of distributions (1.19) satisfy integral identities (1.15) or (1.16). Hence the integral identities (1.15) or (1.16) generalizes the usual integral identities (1.3) to the case of $\delta$-shock wave type solutions.

In the framework of our approach we shall seek a weak asymptotic solution of the Cauchy problems (1.1), (1.13) or (1.9), (1.14) in the form of the smooth ansatz

$$
\begin{aligned}
u(x, t, \varepsilon)= & u_{0}(x, t)+u_{1}(x, t) H_{u}(-x+\phi(t), \varepsilon)+R_{u}(x, t, \varepsilon), \\
v(x, t, \varepsilon)= & v_{0}(x, t)+v_{1}(x, t) H_{v}(-x+\phi(t), \varepsilon) \\
& +e(t) \delta_{v}(-x+\phi(t), \varepsilon)+R_{v}(x, t, \varepsilon),
\end{aligned}
$$

where $u_{k}(x, t), v_{k}(x, t), \quad k=0,1, \quad e(t), \phi(t), R_{u}(x, t, \varepsilon), R_{v}(x, t, \varepsilon)$ are the desired functions. Here

$$
\delta_{v}(x, \varepsilon)=\frac{1}{\varepsilon} \omega_{\delta}(x / \varepsilon)
$$

is a regularization of the $\delta$-function,

$$
H_{j}(x, \varepsilon)=\omega_{0 j}\left(\frac{x}{\varepsilon}\right)=\int_{-\infty}^{x / \varepsilon} \omega_{j}(\eta) d \eta, \quad j=u, v,
$$

are regularizations of the Heaviside function $H(x)$. The mollifiers $\omega_{u}(\eta), \omega_{v}(\eta), \omega_{\delta}(\eta)$ have the following properties: (a) $\omega(\eta) \in C^{\infty}(\mathbb{R})$, (b) $\omega(\eta)$ has a compact support or decreases sufficiently rapidly as $|\eta| \rightarrow \infty$, (c) $\int \omega(\eta) d \eta=1$, (d) $\omega(\eta) \geq 0$.

The corrections $R_{j}(x, t, \varepsilon)$ are functions such that

$$
R_{j}(x, t, \varepsilon)=o_{\mathcal{D}^{\prime}}(1), \quad \frac{\partial R_{j}(x, t, \varepsilon)}{\partial t}=o_{\mathcal{D}^{\prime}}(1), \quad \varepsilon \rightarrow+0,
$$

\footnotetext{
${ }^{1}$ For all cases except system 1.7, one can replace $o_{\mathcal{D}^{\prime}}(1)$ in the right-hand sides of $(1.14)$ by $O_{\mathcal{D}^{\prime}}(\varepsilon)$.
} 
$j=u, v$.

Since the generalized solution of the Cauchy problem is defined as a weak limit (1.19) of a weak asymptotic solution, taking into account estimates (1.23), we see that the corrections $R_{u}(x, t, \varepsilon), R_{v}(x, t, \varepsilon)$ do not make a contribution to the generalized solution of the problem. Otherwise, setting $R_{u}(x, t, \varepsilon)=R_{v}(x, t, \varepsilon)=0$, i.e., without introducing these terms, we cannot solve the Cauchy problem with an arbitrary initial data (see Remarks 2.6, 3.4 below).

\section{Propagation of $\delta$-shocks of system (1.6).}

2.1. Construction of a weak asymptotic solution. Let us consider the propagation of a single $\delta$-shock wave of system (1.6), i.e., consider the Cauchy problem (1.6), (1.13). We assume that the initial data satisfy the condition $u_{1}^{0}(0)>0$.

Since the eigenvalues of the characteristic matrix of system (1.6) are $\lambda_{1}(u, v)=f^{\prime}(u)$, $\lambda_{2}(u, v)=g(u)$, according to (1.17), we assume as in [6]-10], [15] that the following conditions are satisfied

$$
f^{\prime \prime}(u)>0, \quad g^{\prime}(u)>0, \quad f^{\prime}(u) \leq g(u) .
$$

In order to construct a weak asymptotic solution of the Cauchy problem (1.6), (1.13) we choose corrections in the form

$$
\begin{aligned}
& R_{u}(x, t, \varepsilon)=0, \\
& R_{v}(x, t, \varepsilon)=R(t) \frac{1}{\varepsilon} \Omega^{\prime \prime}\left(\frac{-x+\phi(t)}{\varepsilon}\right),
\end{aligned}
$$

where $R(t)$ is the desired function, $\varepsilon^{-3} \Omega^{\prime \prime}(x / \varepsilon)$ is a regularization of the distribution $\delta^{\prime \prime}(x), \Omega(\eta)$ has the properties (a)-(c) (see Subsec. 1.4). Since for any test function $\psi(x) \in \mathcal{D}\left(\mathbb{R}_{x}\right)$ we have

$$
\begin{aligned}
\int \frac{1}{\varepsilon} \Omega^{\prime \prime}\left(\frac{x}{\varepsilon}\right) \psi(x) d x & =\varepsilon^{2} \psi^{\prime \prime}(0) \int \Omega(\xi) d \xi+O\left(\varepsilon^{3}\right), \\
\int \frac{\partial}{\partial x}\left(\frac{1}{\varepsilon} \Omega^{\prime \prime}\left(\frac{x}{\varepsilon}\right)\right) \psi(x) d x & =-\varepsilon^{2} \psi^{\prime \prime \prime}(0) \int \Omega(\xi) d \xi+O\left(\varepsilon^{3}\right),
\end{aligned}
$$

relations (1.23) hold.

Now to calculate some weak asymptotic expansions we use the following lemmas.

Lemma 2.1 ([5, Corollary 1.1]). Let $f(u)$ be a smooth function, let $u_{0}(x, t), u_{1}(x, t)$ be bounded functions. If $u(x, t, \varepsilon)$ is defined by (1.20), (2.2), then

$$
f(u(x, t, \varepsilon))=f\left(u_{+}\right)+[f(u)] H(-x+\phi(t))+O_{\mathcal{D}^{\prime}}(\varepsilon), \quad \varepsilon \rightarrow+0,
$$

where $[f(u(x, t))]=f\left(u_{-}(x, t)\right)-f\left(u_{+}(x, t)\right)$ is a jump in function $f(u(x, t))$ across the discontinuity curve $x=\phi(t), u_{+}=u_{0}, u_{-}=u_{0}+u_{1}$.

Lemma 2.2. Let $g(u)$ be a smooth function, let $u_{k}(x, t), v_{k}(x, t), k=0,1, e(t)$ be bounded functions. If $u(x, t, \varepsilon), v(x, t, \varepsilon)$ are defined by (1.20), (2.2), then

$$
\begin{aligned}
v(x, t, \varepsilon) & g(u(x, t, \varepsilon))=v_{+} g\left(u_{+}\right)+[v g(u)] H(-x+\phi(t)) \\
& +\{e(t) a(t)+R(t) c(t)\} \delta(-x+\phi(t))+O_{\mathcal{D}^{\prime}}(\varepsilon), \quad \varepsilon \rightarrow+0,
\end{aligned}
$$


where $[h(u(x, t), v(x, t))]=h\left(u_{-}(x, t), v_{-}\right)-h\left(u_{+}(x, t), v_{+}(x, t)\right)$ is a jump in function $h(u(x, t), v(x, t))$ across the discontinuity curve $x=\phi(t), u_{+}=u_{0}, v_{+}=v_{0}, u_{-}=u_{0}+u_{1}$, $v_{-}=v_{0}+v_{1}$,

$$
\begin{aligned}
a(t) & =\left.\int g\left(u_{+}(x, t)\left(1-\omega_{0 u}(\eta)\right)+u_{-}(x, t) \omega_{0 u}(\eta)\right)\right|_{x=\phi(t)} \omega_{\delta}(\eta) d \eta, \\
c(t) & =\left.\int g\left(u_{+}(x, t)\left(1-\omega_{0 u}(\eta)\right)+u_{-}(x, t) \omega_{0 u}(\eta)\right)\right|_{x=\phi(t)} \Omega^{\prime \prime}(\eta) d \eta .
\end{aligned}
$$

Proof. Using Lemma 2.1, it is easy to obtain the weak asymptotics

$$
\begin{aligned}
& g(u(x, t, \varepsilon))\left(v_{0}(x, t)+v_{1}(x, t) H_{v}(-x+\phi(t), \varepsilon)\right) \\
& \quad=v_{+} g\left(u_{+}\right)+[v g(u)] H(-x+\phi(t))+O_{\mathcal{D}^{\prime}}(\varepsilon), \quad \varepsilon \rightarrow+0 .
\end{aligned}
$$

Next, after the change of variables $x=-\varepsilon \eta$, we have

$$
\begin{aligned}
& J(\varepsilon)=\left\langleg ( u ( x , t , \varepsilon ) ) \left( e(t) \delta_{v}(-x+\phi(t), \varepsilon)\right.\right. \\
&\left.\left.+R(t) \frac{1}{\varepsilon} \Omega^{\prime \prime}\left(\frac{-x+\phi(t)}{\varepsilon}\right)\right), \psi(x)\right\rangle \\
&=\psi(\phi(t))(e(t) a(t)+R(t) c(t))+O(\varepsilon), \quad \varepsilon \rightarrow+0,
\end{aligned}
$$

for all $\psi(x) \in \mathcal{D}(\mathbb{R})$.

TheOrem 2.3. Let conditions (2.1) be satisfied. Then there exists $T>0$ such that, for $t \in[0, T)$, the Cauchy problem (1.6), (1.13), $u_{1}^{0}(0)>0$ has a weak asymptotic solution (1.20), (2.2) if and only if

$$
\begin{aligned}
L_{11}\left[u_{+}\right] & =0, \quad x>\phi(t), \\
L_{11}\left[u_{-}\right] & =0, \quad x<\phi(t), \\
L_{12}\left[u_{+}, v_{+}\right] & =0, \quad x>\phi(t), \\
L_{12}\left[u_{-}, v_{-}\right] & =0, \quad x<\phi(t), \\
\dot{\phi}(t) & =\left.\frac{[f(u)]}{[u]}\right|_{x=\phi(t)}, \\
\dot{e}(t) & =\left.\left([v g(u)]-[v] \frac{[f(u)]}{[u]}\right)\right|_{x=\phi(t)}, \\
R(t) & =\frac{e(t)}{c(t)}\left(\left.\frac{[f(u)]}{[u]}\right|_{x=\phi(t)}-a(t)\right),
\end{aligned}
$$

where $a(t), c(t) \neq 0$ are defined by (2.3). The initial data for system (2.4) are defined from (1.13), and $\phi(0)=0$.

Proof. Substitute into system (1.6) smooth ansatzs (1.20), asymptotics $f(u(x, t, \varepsilon))$ given by Lemma 2.1 and asymptotics $v(x, t, \varepsilon) g(u(x, t, \varepsilon))$ given by Lemma 2.2, and 
taking into account the estimates (1.23), we obtain up to $O_{\mathcal{D}^{\prime}}(\varepsilon)$ the following relations:

$$
\begin{gathered}
L_{11}[u(x, t, \varepsilon)]=L_{11}\left[u_{+}\right]+\left\{\frac{\partial[u]}{\partial t}+\frac{\partial}{\partial x}[f(u)]\right\} H(-x+\phi(t)) \\
+\{[u] \dot{\phi}(t)-[f(u)]\} \delta(-x+\phi(t))+O_{\mathcal{D}^{\prime}}(\varepsilon), \\
L_{12}[u(x, t, \varepsilon), v(x, t, \varepsilon)]=L_{12}\left[u_{+}, v_{+}\right]+\left\{\frac{\partial[v]}{\partial t}+\frac{\partial}{\partial x}[v g(u)]\right\} H(-x+\phi(t)) \\
+\{[v] \dot{\phi}(t)+\dot{e}(t)-[v g(u)]\} \delta(-x+\phi(t)) \\
+\{e(t) \dot{\phi}(t)-e(t) a(t)-c(t) R(t)\} \delta^{\prime}(-x+\phi(t))+O_{\mathcal{D}^{\prime}}(\varepsilon),
\end{gathered}
$$

where $a(t), c(t)$ are defined by (2.3).

Setting the right-hand side of (2.5), (2.6) equal to zero, we obtain necessary and sufficient conditions for the relations

$$
L_{11}[u(x, t, \varepsilon)]=O_{\mathcal{D}^{\prime}}(\varepsilon), \quad L_{12}[u(x, t, \varepsilon), v(x, t, \varepsilon)]=O_{\mathcal{D}^{\prime}}(\varepsilon),
$$

i.e., the first six equations of system (2.4) and the equation $c(t) R(t)=e(t)(\dot{\phi}(t)-a(t))$, which determines the function $R(t)$.

At the second stage of the proof, we show that system (2.4) is solvable. According to (2.4), two smooth functions $u_{+}$and $u_{-}$, defined on respective domains $\Omega_{t}^{+}=\{x>\phi(t)\}$ and $\Omega_{t}^{-}=\{x<\phi(t)\}$ on either side of the curve $x=\phi(t)$ so that $L_{11}\left[u_{ \pm}\right]=0,(x, t) \in \Omega_{t}^{ \pm}$. The boundary values of $u_{ \pm}$restricted to the curve $x=\phi(t)$ satisfy the Rankine-Hugoniot condition $\dot{\phi}(t)=\left.\frac{[f(u)]}{[u]}\right|_{x=\phi(t)}$. In order to solve this problem, we consider the Cauchy problem $L_{11}[u]=u_{t}+(f(u))_{x}=0, u(x, 0)=u^{0}(x)$. Since $f(u)$ is convex and $u_{1}^{0}(0)>0$, according to the results [18, Ch. 4.2], we extend $u_{+}^{0}(x)=u_{0}^{0}(x)\left(u_{-}^{0}(x)=u_{0}^{0}(x)+u_{1}^{0}(x)\right)$ to $x \leq 0(x \geq 0)$ in a bounded $C^{1}$ fashion and continue to denote the extended functions by $u_{ \pm}^{0}(x)$. By $u_{ \pm}(x, t)$ we denote the $C^{1}$ solutions of the problems

$$
L_{11}[u]=u_{t}+(f(u))_{x}=0, \quad u_{ \pm}(x, 0)=u_{ \pm}^{0}(x)
$$

which exist for small enough time interval $\left[0, T_{1}\right]$ and are determined by integration along characteristics. The functions $u_{ \pm}(x, t)$ determine a two-sheeted covering of the plane $(x, t)$. Next, we define the function $x=\phi(t)$ as a solution of the problem

$$
\dot{\phi}(t)=\left.\frac{f\left(u_{+}(x, t)\right)-f\left(u_{-}(x, t)\right)}{u_{+}(x, t)-u_{-}(x, t)}\right|_{x=\phi(t)}, \quad \phi(0)=0 .
$$

It is clear that there exists a unique function $\phi(t)$ for sufficiently short times $\left[0, T_{2}\right]$. To this end, for $T=\min \left(T_{1}, T_{2}\right)$ we define the shock solution by

$$
u(x, t)= \begin{cases}u_{+}(x, t), & x>\phi(t), \\ u_{-}(x, t), & x<\phi(t) .\end{cases}
$$

Thus the first, second and fifth equations of system (2.4) define a unique solution of the Cauchy problem $L_{11}[u]=u_{t}+(f(u))_{x}=0, u(x, 0)=u^{0}(x)$ for $t \in[0, T)$.

Solving this problem, we obtain $u(x, t), \phi(t)$. Then substituting these functions into (2.4), we obtain $V(x, t)=v_{0}(x, t)+v_{1}(x, t) H(-x+\phi(t)), e(t)$, and $v(x, t)=V(x, t)+$ $e(t) \delta(-x+\phi(t))$. 
It is clear that functions $\omega_{0 u}(\xi), \Omega^{\prime \prime}(\xi)$ can be chosen such that $\int \omega_{u}(\eta) \Omega^{\prime}(\eta) d \eta>0$. Taking into account that in view of condition (2.1) $g^{\prime}(u)>0, u_{1}(\phi(t), t)>0$, and integrating by parts, we have

$$
c(t)=-\left.\int g^{\prime}\left(u_{0}(x, t)+u_{1}(x, t) \omega_{0 u}(\eta)\right) u_{1}(x, t)\right|_{x=\phi(t)} \omega_{u}(\eta) \Omega^{\prime}(\eta) d \eta \neq 0 .
$$

So for any functions $u_{ \pm}(x, t), e(t), \phi(t), t \in[0, T)$, there exists the function $R(t)$, which is determined by the last relation of (2.4).

2.2. Construction of a generalized solution. Using a weak asymptotic solution constructed by Theorem 2.3 we obtain a generalized solution of the Cauchy problem (1.6), (1.13) as a weak limit (1.19).

Theorem 2.4. Let $u_{1}^{0}(0)>0$ and conditions (2.1) be satisfied. Then, for $t \in[0, T)$, where $T>0$ is given by Theorem 2.3, the Cauchy problem (1.6), (1.13), $u_{1}^{0}(0)>0$ has a unique generalized solution

$$
\begin{aligned}
& u(x, t)=u_{+}(x, t)+[u(x, t)] H(-x+\phi(t)), \\
& v(x, t)=v_{+}(x, t)+[v(x, t)] H(-x+\phi(t))+e(t) \delta(-x+\phi(t)),
\end{aligned}
$$

which satisfies the integral identities; cf. (1.15):

$$
\begin{aligned}
\int_{0}^{T} \int\left(u \varphi_{t}+f(u) \varphi_{x}\right) d x d t+\int u^{0}(x) \varphi(x, 0) d x & =0 \\
\int_{0}^{T} \int\left(\varphi_{t}+g(u) \varphi_{x}\right) V d x d t+\int V^{0}(x) \varphi(x, 0) d x & \\
+\int_{\Gamma} e(x, t) \frac{\partial \varphi(x, t)}{\partial \mathbf{l}} d l+e^{0} \varphi(0,0) & =0
\end{aligned}
$$

where $\Gamma=\{(x, t): x=\phi(t), t \in[0, T)\}$, and

$$
\int_{\Gamma} e(x, t) \frac{\partial \varphi(x, t)}{\partial \mathbf{l}} d l=\int_{0}^{T} e(t)\left(\varphi_{t}(\phi(t), t)+\dot{\phi}(t) \varphi_{x}(\phi(t), t)\right) d t,
$$

$V(x, t)=v_{+}+[v] H(-x+\phi(t))$ and functions $u_{ \pm}(x, t), v_{ \pm}(x, t), \phi(t), e(t)$ are determined by the system

$$
\begin{aligned}
L_{11}\left[u_{+}\right] & =0, \quad x>\phi(t), \\
L_{11}\left[u_{-}\right] & =0, \quad x<\phi(t), \\
L_{12}\left[u_{+}, v_{+}\right] & =0, \quad x>\phi(t), \\
L_{12}\left[u_{-}, v_{-}\right] & =0, \quad x<\phi(t), \\
\dot{\phi}(t) & =\left.\frac{[f(u)]}{[u]}\right|_{x=\phi(t)}, \\
\dot{e}(t) & =\left.\left([v g(u)]-[v] \frac{[f(u)]}{[u]}\right)\right|_{x=\phi(t)} .
\end{aligned}
$$

Proof. By substituting the last relation (2.4), which determines $R(t)$, into the relation $v(x, t, \varepsilon) g(u(x, t, \varepsilon))$ given by Lemma 2.2 , we obtain

$$
\begin{aligned}
& v(x, t, \varepsilon) g(u(x, t, \varepsilon))=v_{0} g\left(u_{0}\right)+[v g(u)] H(-x+\phi(t)) \\
& +\left.e(t) \frac{[f(u)]}{[u]}\right|_{x=\phi(t)} \delta(-x+\phi(t))+O_{\mathcal{D}^{\prime}}(\varepsilon), \quad \varepsilon \rightarrow+0,
\end{aligned}
$$


By Theorem 2.3 we have the following estimates:

$$
L_{11}[u(x, t, \varepsilon)]=O_{\mathcal{D}^{\prime}}(\varepsilon), \quad L_{12}[u(x, t, \varepsilon), v(x, t, \varepsilon)]=O_{\mathcal{D}^{\prime}}(\varepsilon) .
$$

Let us apply the left-hand and right-hand sides of these relations to an arbitrary test function $\varphi(x, t) \in \mathcal{D}(\mathbb{R} \times[0, T))$. Then integrating by parts, we obtain

$$
\begin{gathered}
\int_{0}^{T} \int\left(u(x, t, \varepsilon) \varphi_{t}(x, t)+f(u(x, t, \varepsilon)) \varphi_{x}(x, t)\right) d x d t \\
+\int u(x, 0, \varepsilon) \varphi(x, 0) d x=O(\varepsilon), \\
\int_{0}^{T} \int\left(\varphi_{t}(x, t)+g(u(x, t, \varepsilon)) \varphi_{x}(x, t)\right) v(x, t, \varepsilon) d x d t \\
+\int v(x, 0, \varepsilon) \varphi(x, 0) d x=O(\varepsilon), \quad \varepsilon \rightarrow+0 .
\end{gathered}
$$

Passing to the limit as $\varepsilon \rightarrow+0$ and taking into account Lemmas 2.1, 2.2, relation (2.9), and the fact that

$$
\begin{aligned}
\lim _{\varepsilon \rightarrow+0} \int_{0}^{T} \int e(t) \delta_{v}(-x+\phi(t), \varepsilon) \varphi(x, t) d x d t & =\int_{0}^{T} e(t) \varphi(\phi(t), t) d t \\
\lim _{\varepsilon \rightarrow+0} \int e(0) \delta_{v}(-x, \varepsilon) \varphi(x, 0) d x & =e(0) \varphi(0,0)
\end{aligned}
$$

we obtain the integral identities (2.7).

In view of above arguments in Theorem 2.4, the Cauchy problem (1.6), (1.13), $u_{1}^{0}(0)>$ 0 has a unique generalized solution.

Here the right-hand side of the sixth equation of system (2.4)

$$
\dot{e}(t)=\left.\left([v g(u)]-[v] \frac{[f(u)]}{[u]}\right)\right|_{x=\phi(t)}
$$

is the so-called Rankine-Hugoniot deficit.

If the initial data (1.13) are piecewise constant and $u_{1}^{0}=u_{1}=[u]>0$, then from Theorem 2.4 we obtain the following corollary.

Corollary 2.5. For $t \in[0, \infty)$, the Cauchy problem (1.6), (1.13), (2.1), with piecewise constant initial data has a unique generalized solution

$$
\begin{aligned}
& u(x, t)=u_{+}+[u] H(-x+\phi(t)) \\
& v(x, t)=v_{+}+[v] H(-x+\phi(t))+e(t) \delta(-x+\phi(t))
\end{aligned}
$$

which satisfies the integral identities (2.7), where

$$
\begin{aligned}
\phi(t) & =\frac{[f(u)]}{[u]} t \\
e(t) & =e^{0}+\left([v g(u)]-\frac{[f(u)]}{[u]}[v]\right) t .
\end{aligned}
$$

REMARK 2.6. To find a generalized solution of the Cauchy problem (1.6), (1.13), we construct a weak asymptotic solution (1.20), (2.2) of the problem, where the functions 
$u_{ \pm}(x, t), v_{ \pm}(x, t), e(t), \phi(t)$, and the functions $\omega_{0 u}(\eta), \omega_{\delta}(\eta), \Omega^{\prime \prime}(\eta)$ and $R(t)$ are determined by system (2.4). Without introducing correction (2.2), i.e., setting $R(t)=0$, we derive from (2.4) that we can solve the Cauchy problem only if the relation

$$
\begin{aligned}
& \left.\frac{[f(u(x, t))]}{[u(x, t)]}\right|_{x=\phi(t)} \\
& \quad=\left.\int g\left(\omega_{0 u}(\eta) u_{-}(x, t)+\left(1-\omega_{0 u}(\eta)\right) u_{+}(x, t)\right)\right|_{x=\phi(t)} \omega_{\delta}(\eta) d \eta
\end{aligned}
$$

holds. In the general case, this relation makes the Cauchy problem (1.6), (1.13) overdetermined, i.e., we cannot solve this Cauchy problem with an arbitrary jump.

Nevertheless, in the case of the piecewise constant initial data (1.13), in view of conditions (1.17) and (2.1), we can choose the mollifiers $\omega_{u}, \omega_{\delta}$ in (2.3) such that the above relation holds, i.e.,

$$
\int g\left(\omega_{0 u}(\eta) u_{-}+\left(1-\omega_{0 u}(\eta)\right) u_{+}\right) \omega_{\delta}(\eta) d \eta=\frac{[f(u)]}{[u]} .
$$

Thus in the case of the piecewise constant initial data, we can set $R(t)=0$.

\section{Propagation of $\delta$-shocks of system (1.7).}

3.1. Construction of a weak asymptotic solution. Let us consider the Cauchy problem (1.7), (1.13). Since the eigenvalues of the characteristic matrix of system (1.7) are $\lambda_{1}(u)=$ $u-1, \lambda_{2}(u)=u+1$, the "overcompression" condition has the form

$$
\lambda_{1}\left(u_{+}\right) \leq \lambda_{2}\left(u_{+}\right) \leq \dot{\phi}(t) \leq \lambda_{1}\left(u_{-}\right) \leq \lambda_{2}\left(u_{-}\right) .
$$

We choose corrections $R(x, t, \varepsilon)$ in the form

$$
\begin{aligned}
& R_{u}(x, t, \varepsilon)=P(t) \frac{1}{\sqrt{\varepsilon}} \Omega_{P}\left(\frac{-x+\phi(t)}{\varepsilon}\right)+Q(t) \frac{1}{\sqrt[3]{\varepsilon}} \Omega_{Q}\left(\frac{-x+\phi(t)}{\varepsilon}\right) \\
& R_{v}(x, t, \varepsilon)=0
\end{aligned}
$$

where $P(t), Q(t)$ are the desired functions, $\frac{1}{\varepsilon} \Omega_{P}^{2}(x / \varepsilon), \frac{1}{\varepsilon} \Omega_{Q}^{3}(x / \varepsilon)$ are regularizations (1.21) of the delta function, mollifiers $\Omega_{P}(\eta), \Omega_{Q}(\eta)$ have properties (a)-(c) (see Subsec. 1.4). It is clear that estimates (1.23) hold.

In addition to (3.2), we can choose mollifiers $\Omega_{P}(\eta), \Omega_{Q}(\eta)$ such that

$$
\int \Omega_{P}^{3}(\eta) d \eta=0, \quad \int \Omega_{P}^{2}(\eta) \Omega_{Q}(\eta) d \eta=0, \quad \int \Omega_{P}(\eta) \Omega_{Q}^{2}(\eta) d \eta=0 .
$$

For example, we can choose $\Omega_{P}(\eta)=\eta e^{-\eta^{2}}, \Omega_{Q}(\eta)=\left(1-2 \eta^{2}\right) e^{-\eta^{2}}$. This example was proposed by V. I. Polischook.

Theorem 3.1. Let

$$
u_{+}^{0}(0)+1 \leq\left.\frac{\left[\left(u^{0}\right)^{2}\right]-\left[v^{0}\right]}{\left[u^{0}\right]}\right|_{x=0} \leq u_{-}^{0}(0)-1
$$


$\left(u_{+}^{0}=u_{0}^{0}, u_{-}^{0}=u_{0}^{0}+u_{1}^{0}\right)$ then there exists $T>0$ such that, for $t \in[0, T)$, the Cauchy problem (1.7), (1.13), has a weak asymptotic solution (1.20), (3.2), (3.3) if and only if

$$
\begin{aligned}
L_{21}\left[u_{+}, v_{+}\right] & =0, \quad x>\phi(t), \\
L_{21}\left[u_{-}, v_{-}\right] & =0, \quad x<\phi(t), \\
L_{22}\left[u_{+}, v_{+}\right] & =0, \quad x>\phi(t), \\
L_{22}\left[u_{-}, v_{-}\right] & =0, \quad x<\phi(t), \\
\dot{\phi}(t) & =\left.\frac{\left[u^{2}\right]-[v]}{[u]}\right|_{x=\phi(t)}, \\
\dot{e}(t) & =\left.\left(\frac{\left[u^{3}\right]}{3}-[u]-[v] \frac{\left.u^{2}\right]-[v]}{[u]}\right)\right|_{x=\phi(t)}, \\
P(t) & =\sqrt{\frac{e(t)}{a}} \\
Q(t) & =\left.\sqrt[3]{\frac{3 e(t)}{c}\left(\frac{\left[u^{2}\right]-[v]}{[u]}-\left(1-\frac{b}{a}\right) u_{+}-\frac{b}{a} u_{-}\right)}\right|_{x=\phi(t)}
\end{aligned}
$$

where $u_{+}=u_{0}, v_{+}=v_{0}, u_{-}=u_{0}+u_{1}, v_{-}=v_{0}+v_{1}$,

$$
\begin{aligned}
a & =\int \Omega_{P}^{2}(\eta) d \eta>0, \\
b & =\int \omega_{0 u}(\eta) \Omega_{P}^{2}(\eta) d \eta, \\
c & =\int \Omega_{Q}^{3}(\eta) d \eta \neq 0 .
\end{aligned}
$$

The initial data for system (3.5) are defined from (1.13), and $\phi(0)=0$.

Proof. Let $H(x, \varepsilon)=\omega_{0}(x / \varepsilon)$ be a regularization (1.22) of the Heaviside function, and let $\delta_{k}(x, \varepsilon)=\varepsilon^{-1} \omega_{k}(x / \varepsilon), \quad k=1,2$ be regularizations (1.21) of the delta function. Using Lemmas 2.1, 2.2, we obtain the following relations:

$$
\begin{aligned}
(H(x, \varepsilon))^{r} & =H(x)+O_{\mathcal{D}^{\prime}}(\varepsilon), \\
\delta_{1}(x, \varepsilon)\left(\omega_{2}\left(\frac{x}{\varepsilon}\right)\right)^{r} & =A_{r} \delta(x)+O_{\mathcal{D}^{\prime}}(\varepsilon), \\
\delta_{1}(x, \varepsilon)(H(x, \varepsilon))^{r} & =B_{r} \delta(x)+O_{\mathcal{D}^{\prime}}(\varepsilon), \quad \varepsilon \rightarrow+0,
\end{aligned}
$$

where $A_{r}=\int \omega_{1}(\eta) \omega_{2}^{r}(\eta) d \eta, B_{r}=\int \omega_{0}^{r}(\eta) \omega(\eta) d \eta, \quad r=1,2, \ldots$

With the help of (3.7), (3.3), (3.6), one can calculate the following weak asymptotics

$$
\begin{aligned}
R^{2}(x, t, \varepsilon) & =a P^{2}(t) \delta(-x+\phi(t))+o_{\mathcal{D}^{\prime}}(1), \\
R^{3}(x, t, \varepsilon) & =c Q^{3}(t) \delta(-x+\phi(t))+o_{\mathcal{D}^{\prime}}(1), \\
H(-x+\phi(t), \varepsilon) R^{2}(x, t, \varepsilon) & =b P^{2}(t) \delta(-x+\phi(t))+o_{\mathcal{D}^{\prime}}(1),
\end{aligned}
$$

where $a, b, c$ are defined by (3.6). Next, using relations (3.7), we find

$$
\begin{aligned}
(u(x, t, \varepsilon))^{2}= & u_{+}^{2}+\left[u^{2}\right] H(-x+\phi(t))+R^{2}(x, t, \varepsilon)+o_{\mathcal{D}^{\prime}}(1), \\
(u(x, t, \varepsilon))^{3}= & u_{+}^{3}+\left[u^{3}\right] H(-x+\phi(t))+3\left(u_{+}+[u] H(-x+\phi(t), \varepsilon)\right) \\
& \times R^{2}(x, t, \varepsilon)+R^{3}(x, t, \varepsilon)+o_{\mathcal{D}^{\prime}}(1) .
\end{aligned}
$$


Relations (3.8) and (3.9) thus imply with accuracy $o_{\mathcal{D}^{\prime}}(1)$ the following relations:

$$
\begin{aligned}
(u(x, t, \varepsilon))^{2}= & u_{+}^{2}+\left[u^{2}\right] H(-x+\phi(t)) \\
& +a P^{2}(t) \delta(-x+\phi(t))+o_{\mathcal{D}^{\prime}}(1), \\
(u(x, t, \varepsilon))^{3}= & u_{+}^{3}+\left[u^{3}\right] H(-x+\phi(t)) \\
& +\left(3\left((a-b) u_{+}+b u_{-}\right) P^{2}(t)+c Q^{3}(t)\right) \delta(-x+\phi(t)) \\
& +o_{\mathcal{D}^{\prime}}(1), \quad \varepsilon \rightarrow+0 .
\end{aligned}
$$

Substituting (1.20), (3.2), (3.10) into the left-hand side of system (1.7), we obtain, up to $o_{\mathcal{D}^{\prime}}(1)$,

$$
\begin{aligned}
L_{21}[u(x, t, \varepsilon) & , v(x, t, \varepsilon)]=L_{21}\left[u_{+}, v_{+}\right] \\
+ & \left\{\frac{\partial[u]}{\partial t}+\frac{\partial}{\partial x}\left[u^{2}-v\right]\right\} H(-x+\phi(t)) \\
+ & \left\{[u] \dot{\phi}(t)-\left[u^{2}-v\right]\right\} \delta(-x+\phi(t)) \\
& +\left\{e(t)-a P^{2}(t)\right\} \delta^{\prime}(-x+\phi(t))+o_{\mathcal{D}^{\prime}}(1), \\
L_{22}[u(x, t, \varepsilon), & v(x, t, \varepsilon)]=L_{22}\left[u_{+}, v_{+}\right] \\
+ & \left\{\frac{\partial[v]}{\partial t}+\frac{\partial}{\partial x}\left[\frac{u^{3}}{3}-u\right]\right\} H(-x+\phi(t)) \\
+ & \left\{[v] \dot{\phi}(t)+\dot{e}(t)-\left[\frac{u^{3}}{3}-u\right]\right\} \delta(-x+\phi(t)) \\
+\left\{e(t) \dot{\phi}(t)-\left((a-b) u_{+}+b u-\right) P^{2}(t)\right. & \left.\quad-\frac{c}{3} Q^{3}(t)\right\} \delta^{\prime}(-x+\phi(t))+o_{\mathcal{D}^{\prime}}(1), \quad \varepsilon \rightarrow+0 .
\end{aligned}
$$

Setting the left-hand side of (3.11), (3.12) equal to zero, we obtain systems (3.5).

Now we consider the Cauchy problem

$$
\begin{aligned}
& L_{21}[u, V]=0, \quad u(x, 0)=u^{0}(x), \\
& L_{22}[u, V]=0, \quad V(x, 0)=V^{0}(x)=v_{0}^{0}(x)+v_{1}^{0}(x) H(-x),
\end{aligned}
$$

assuming that condition (3.4) holds. The last condition means that $\left(u^{0}(x), V^{0}(x)\right)$ is entropy initial data. To solve this Cauchy problem we repeat the second part of the proof of Theorem 2.3 almost word for word. Namely, according to [18, Ch. 4.2], we extend a pair of functions

$$
\begin{aligned}
\left(u_{+}^{0}(x)=u_{0}^{0}(x), V_{+}^{0}(x)=v_{0}^{0}(x)\right), & x \leq 0, \\
\left(u_{-}^{0}(x)=u_{0}^{0}(x)+u_{1}^{0}(x), V_{-}^{0}(x)=v_{0}^{0}(x)+v_{1}^{0}(x)\right), & x \geq 0,
\end{aligned}
$$

in a bounded $C^{1}$ and denote the extended pair by $\left(u_{ \pm}^{0}(x), V_{ \pm}^{0}(x)\right)$. According to $[18$, Ch. 2.1], [21, Ch. I, §8], for small enough time interval $\left[0, T_{1}\right]$ the Cauchy problems

$$
\begin{array}{ll}
L_{21}[u, V]=0, & u_{ \pm}(x, 0)=u_{ \pm}^{0}(x), \\
L_{22}[u, V]=0, & V_{ \pm}(x, 0)=V_{ \pm}^{0}(x) .
\end{array}
$$

have solutions $\left(u_{ \pm}(x, t), V_{ \pm}(x, t)\right) \in C^{1}$, respectively. The pair $\left(u_{ \pm}(x, t), V_{ \pm}(x, t)\right)$ determines a two-sheeted covering of the plane $(x, t)$. In view of the overcompression condition 
(3.1), (3.4), there exists a unique solution $x=\phi(t)$ of the problem

$$
\dot{\phi}(t)=\left.\frac{f\left(u_{-}(x, t)\right)-f\left(u_{+}(x, t)\right)-V_{-}(x, t)+V_{+}(x, t)}{u_{-}(x, t)-u_{+}(x, t)}\right|_{x=\phi(t)}, \quad \phi(0)=0
$$

for sufficiently short times $\left[0, T_{2}\right]$. Thus we construct the unique shock solution

$$
(u(x, t), V(x, t))= \begin{cases}\left(u_{+}(x, t), V_{+}(x, t)\right), & x>\phi(t), \\ \left(u_{-}(x, t), V_{-}(x, t)\right), & x<\phi(t)\end{cases}
$$

of the Cauchy problem (3.13) for $T=\min \left(T_{1}, T_{2}\right)$, which is determined by the first five equations of system (3.5).

Solving this problem, we obtain $u(x, t), V(x, t), \phi(t)$. Then substituting these functions into the sixth equation of system (3.5), we obtain $e(t), v(x, t)=V(x, t)+e(t) \delta(-x+$ $\phi(t))$. Next, for any functions $u_{ \pm}(x, t), v_{ \pm}(x, t), e(t), \phi(t), t \in[0, T)$, there exist functions $P(t), Q(t)$ which are determined by the seventh and eighth equations of (3.5).

3.2. Construction of a generalized solution. Using a weak asymptotic solution of the problem, just as above in Subsec. 2.2, we can prove the following theorem.

Theorem 3.2. There exists $T>0$ such that the Cauchy problem (1.7), (1.13), (3.4) for $t \in[0, T)$ has a unique generalized solution

$$
\begin{aligned}
& u(x, t)=u_{+}(x, t)+[u(x, t)] H(-x+\phi(t)), \\
& v(x, t)=v_{+}(x, t)+[v(x, t)] H(-x+\phi(t))+e(t) \delta(-x+\phi(t)),
\end{aligned}
$$

which satisfies the integral identities (1.15):

$$
\begin{aligned}
\int_{0}^{T} \int\left(u \varphi_{t}+\left(u^{2}-V\right) \varphi_{x}\right) d x d t+\int u^{0}(x) \varphi(x, 0) d x & =0 \\
\int_{0}^{T} \int\left(V \varphi_{t}+\left(\frac{1}{3} u^{3}-u\right) \varphi_{x}\right) d x d t+\int^{0} V^{0}(x) \varphi(x, 0) d x & \\
+\int_{\Gamma} e(x, t) \frac{\partial \varphi(x, t)}{\partial \mathbf{l}} d l+e^{0} \varphi(0,0) & =0
\end{aligned}
$$

where $\Gamma=\{(x, t): x=\phi(t), t \in[0, T)\}$,

$$
\int_{\Gamma} e(x, t) \frac{\partial \varphi(x, t)}{\partial \mathbf{l}} d l=\int_{0}^{T} e(t)\left(\varphi_{t}(\phi(t), t)+\dot{\phi}(t) \varphi_{x}(\phi(t), t)\right) d t,
$$

$V(x, t)=v_{0}(x, t)+v_{1}(x, t) H(-x+\phi(t))$, and functions $u_{ \pm}(x, t), v_{ \pm}(x, t), \phi(t), e(t)$ are defined by the system

$$
\begin{aligned}
L_{21}\left[u_{+}, v_{+}\right] & =0, \quad x>\phi(t), \\
L_{21}\left[u_{-}, v_{-}\right] & =0, \quad x<\phi(t), \\
L_{22}\left[u_{+}, v_{+}\right] & =0, \quad x>\phi(t), \\
L_{22}\left[u_{-}, v_{-}\right] & =0, \quad x<\phi(t), \\
\dot{\phi}(t) & =\left.\frac{\left[u^{2}\right]-[v]}{[u]}\right|_{x=\phi(t)}, \\
\dot{e}(t) & =\left.\left(\frac{\left[u^{3}\right]}{3}-[u]-[v] \frac{\left[u^{2}\right]-[v]}{[u]}\right)\right|_{x=\phi(t)} .
\end{aligned}
$$


Proof. Using (1.6), (3.5), (3.10), we obtain the following relations:

$$
\begin{gathered}
(u(x, t, \varepsilon))^{2}-v(x, t, \varepsilon)=u_{0}^{2}-v_{0}+\left[u^{2}-v\right] H(-x+\phi(t))+o_{\mathcal{D}^{\prime}}(1), \\
\frac{1}{3}(u(x, t, \varepsilon))^{3}-u(x, t, \varepsilon)=\frac{1}{3} u_{0}^{3}-u_{0}+\left[\frac{1}{3} u^{3}-u\right] H(-x+\phi(t)) \\
+e(t) \frac{\left[u^{2}\right]}{[u]} \delta(-x+\phi(t))+o_{\mathcal{D}^{\prime}}(1), \quad \varepsilon \rightarrow+0 .
\end{gathered}
$$

By Theorem 3.1 we have

$$
L_{21}[u(x, t, \varepsilon), v(x, t, \varepsilon)]=O_{\mathcal{D}^{\prime}}(\varepsilon), \quad L_{22}[u(x, t, \varepsilon), v(x, t, \varepsilon)]=O_{\mathcal{D}^{\prime}}(\varepsilon) .
$$

Substituting (3.16), (3.17) into the above relations and repeating the proof of Theorem 2.4 almost word for word, we obtain the proof of our assertion.

The right-hand side of the sixth equation of system (3.5),

$$
\dot{e}(t)=\left.\left(\frac{\left[u^{3}\right]}{3}-[u]-[v] \frac{\left[u^{2}\right]-[v]}{[u]}\right)\right|_{x=\phi(t)}
$$

is the Rankine-Hugoniot deficit.

Corollary $3.3([24)$. The Cauchy problem (1.7), (1.13), (3.4), where initial data are piecewise constant, for $t \in[0, \infty)$ has a unique generalized solution

$$
\begin{aligned}
& u(x, t)=u_{+}+[u] H(-x+\phi(t)), \\
& v(x, t)=v_{+}+[v] H(-x+\phi(t))+e(t) \delta(-x+\phi(t)),
\end{aligned}
$$

which satisfies the integral identities (3.14), where

$$
\begin{aligned}
& \phi(t)=\frac{\left[u^{2}\right]-[v]}{[u]} t \\
& e(t)=e^{0}+\left(\frac{\left[u^{3}\right]}{3}-[u]-[v] \frac{\left[u^{2}\right]-[v]}{[u]}\right) t .
\end{aligned}
$$

Moreover, if $e^{0}=0$, the Rankine-Hugoniot deficit is positive:

$$
\dot{e}(t)=\frac{\left[u^{3}\right]}{3}-[u]-[v] \frac{\left[u^{2}\right]-[v]}{[u]}>0 .
$$

For the case of piecewise constant initial data and $e^{0}=0$, positiveness of $\dot{e}(t)$ follows from the seventh equation of (3.5) and (3.6). This fact coincides to the statement in [14].

REMARK 3.4. We construct a generalized solution of the Cauchy problem (1.7), (1.13), (3.4) as the weak limit of a weak asymptotic solution of the problem given by Theorem 3.1. Here the functions $u_{ \pm}(x, t), v_{ \pm}(x, t), \phi(t), e(t)$, and $\omega_{0 u}(\eta), \Omega_{P}(\eta), \Omega_{Q}(\eta), P(t), Q(t)$ are determined by relations (3.3), (3.5), (3.6). But in view of estimate (1.23) and formulas (3.16), (3.17), the generalized solution of the Cauchy problem does not depend on correction functions $P(t), Q(t)$. However, in the general case, according to (3.5), without introducing corrections (3.2), we cannot solve the Cauchy problem, which admits $\delta$-shocks.

Indeed, setting in (3.2) $Q(t) \equiv 0$, according to the eighth equation of (2.3), we can construct a weak asymptotic solution of the Cauchy problem only if the following relation

$$
\left.\frac{\left[u^{2}\right]-[v]}{[u]}\right|_{x=\phi(t)}=\left.\left(\left(1-\frac{b}{a}\right) u_{+}+\frac{b}{a} u_{-}\right)\right|_{x=\phi(t)},
$$


holds, where the constants $a, b$ are defined by (3.6). This relation can be rewritten as

$$
\left.\frac{u_{+}(x, t)-\frac{[v(x, t)]}{[u(x, t)]}}{[u(x, t)]}\right|_{x=\phi(t)}=\left.\frac{\dot{\phi}(t)-u_{-}(x, t)}{[u(x, t)]}\right|_{x=\phi(t)}=\frac{b-a}{a} .
$$

In the general case, relation (3.18) makes the Cauchy problem overdetermined, i.e., we cannot solve this Cauchy problem with an arbitrary jump.

In [14, in the framework of the Colombeau theory, an approximate solution of the Cauchy problem (1.7), (1.13) with piecewise constant initial data was constructed. In this paper the correction term of the type

$$
P(t) \frac{1}{\sqrt{\varepsilon}} \Omega_{P}\left(\frac{-x+\phi(t)}{\varepsilon}\right)
$$

was used, and the parameter $a=\int \Omega_{P}^{2}(\eta) d \eta$ was set to be 1 . In this case (3.18) implies the relation

$$
\frac{u_{-}-\dot{\phi}(t)}{[u]}=1-b
$$

where $b=\int \omega_{0 u}(\eta) \Omega_{P}^{2}(\eta) d \eta, a=\int \Omega_{P}^{2}(\eta) d \eta=1$. Thus we have

$$
0<1-b=\int\left(1-\omega_{0 u}(\eta)\right) \Omega_{P}^{2}(\eta) d \eta<1
$$

Here relation (3.19) coincides with the second relation of [14, Proposition 2] and the last inequality coincides with the statement of Lemma 1 from [14. However, in this case, according to [14, Proposition 2], relation (3.19) still leaves one degree of freedom, to connect $u_{-}=u_{0}+u_{1}$ and $u_{+}=u_{0}$. Thus, according to [14], in the case of the piecewise constant initial data (1.13), we can construct a weak asymptotic solution of the Cauchy problem setting in (3.2) $Q(t) \equiv 0$.

If we set in (3.2) $P(t) \equiv 0$ then, according to the seventh equation of (3.5), $e(t)=0$, i.e.,

$$
\left.\left(\frac{\left[u^{3}\right]}{3}-[u]-[v] \frac{\left[u^{2}\right]-[v]}{[u]}\right)\right|_{x=\phi(t)}=0 .
$$

Thus the Cauchy problem (1.7), (1.13), cannot have $\delta$-shock solutions and can be solved only if relation (3.20) holds. Thus, without introducing $\delta$-shocks, we have the overdetermined Cauchy problem.

\section{Propagation of $\delta$-shocks of zero-pressure gas dynamics system.}

4.1. Construction of a weak asymptotic solution. As was already mentioned above in Subsec. 1.2, we consider the Cauchy problem (1.9) with initial data of the form (1.14) instead of (1.13).

System (1.9) has a double eigenvalue $\lambda_{1}(u)=\lambda_{2}(u)=u$. In this case the entropy condition (1.17) is

$$
u_{+} \leq \dot{\phi}(t) \leq u_{-} .
$$

We choose corrections in the form

$$
\begin{aligned}
& R_{u}(x, t, \varepsilon)=Q(t) \Omega^{\prime}\left(\frac{-x+\phi(t)}{\varepsilon}\right), \\
& R_{v}(x, t, \varepsilon)=R(t) \frac{1}{\varepsilon} \widetilde{\Omega}^{\prime \prime}\left(\frac{-x+\phi(t)}{\varepsilon}\right),
\end{aligned}
$$


where $Q(t), R(t)$ are desired functions, $\varepsilon^{-1} \Omega(x / \varepsilon)$ and $\varepsilon^{-1} \widetilde{\Omega}(x / \varepsilon)$ are regularizations of the delta function, $\Omega(\eta)$ and $\widetilde{\Omega}(\eta)$ have the properties (a)-(c) (see Sec. (1). Thus, relations (1.23) hold.

In addition, we choose mollifiers $\Omega(\eta), \widetilde{\Omega}(\eta)$ such that

$$
\begin{aligned}
b_{1}=\int \omega_{\delta}(\eta) \Omega^{\prime}(\eta) d \eta & \neq 0, \\
\int \omega_{0 u}(\eta) \widetilde{\Omega}^{\prime \prime}(\eta) d \eta & =0, \\
c_{2}=\int \omega_{0 u}^{2}(\eta) \widetilde{\Omega}^{\prime \prime}(\eta) d \eta & \neq 0, \\
\int \omega_{0 u}(\eta) \Omega^{\prime}(\eta) \widetilde{\Omega}^{\prime \prime}(\eta) d \eta & =0, \\
\int \Omega^{\prime}(\eta) \widetilde{\Omega}^{\prime \prime}(\eta) d \eta & =0 .
\end{aligned}
$$

It is clear that for any mollifiers $\omega_{\delta}(\eta), \omega_{0 u}(\eta)$, one can choose mollifiers $\Omega(\eta), \widetilde{\Omega}(\eta)$ such that system (4.3) is solvable. For example, we can choose an even mollifier $\widetilde{\Omega}(\eta)$ such that the second and third relations (4.3) hold. Moreover, we assume that $\Omega(\eta)$, $\widetilde{\Omega}(\eta)$ such that $\operatorname{supp}\left(\Omega^{\prime}\right) \cap \operatorname{supp}\left(\widetilde{\Omega}^{\prime \prime}\right)=\emptyset$, i.e., the fourth and fifth relations (4.3) hold. In addition, we assume that the mollifier $\Omega(\eta)$ is such that the plot of the function $\Omega^{\prime}(\eta)$ is similar to the plot of an odd function. Then the first relation (4.3) holds.

Theorem 4.1. Let inequality (4.1) hold for $t=0$, then there exists $T>0$ such that the Cauchy problem (1.9), (1.14) for $t \in[0, T$ ) has a weak asymptotic solution (1.20), (4.2) if and only if

$$
\begin{aligned}
L_{31}\left[u_{+}, v_{+}\right]= & 0, \quad x>\phi(t), \\
L_{31}\left[u_{-}, v_{-}\right]= & 0, \quad x<\phi(t), \\
L_{32}\left[u_{+}, v_{+}\right]= & 0, \quad x>\phi(t), \\
L_{32}\left[u_{-}, v_{-}\right]= & 0, \quad x<\phi(t), \\
\dot{e}(t)= & \left.([u v]-[v] \dot{\phi}(t))\right|_{x=\phi(t)}, \\
\frac{d(e(t) \dot{\phi}(t))}{d t}= & \left.\left(\left[u^{2} v\right]-[u v] \dot{\phi}(t)\right)\right|_{x=\phi(t)}, \\
Q(t)= & \left.\frac{\dot{\phi}(t)-u_{0}-a_{1} u_{1}}{b_{1}}\right|_{x=\phi(t)}, \\
R(t)= & \frac{e(t)}{u_{1}^{2} c_{2}}\left\{(\dot{\phi}(t))^{2}-\left(u_{0}^{2}+2 u_{0} u_{1} a_{1}+u_{1}^{2} a_{2}\right.\right. \\
& \left.\left.+Q^{2}(t) b_{2}+2 Q(t)\left(u_{0} b_{1}+u_{1} c_{1}\right)\right)\right\}\left.\right|_{x=\phi(t)},
\end{aligned}
$$


where the initial data for system (4.4) are defined from (1.14), $\phi(0)=0$, and

$$
\begin{aligned}
a_{k} & =\int\left(\omega_{0 u}(\eta)\right)^{k} \omega_{\delta}(\eta) d \eta, \quad k=1,2, \\
b_{2} & =\int\left(\Omega^{\prime}(\eta)\right)^{2} \omega_{\delta}(\eta) d \eta, \\
c_{1} & =\int \omega_{0 u}(\eta) \Omega^{\prime}(\eta) \omega_{\delta}(\eta) d \eta, \\
c_{2} & =\int \omega_{0 u}^{2}(\eta) \widetilde{\Omega}^{\prime \prime}(\eta) d \eta .
\end{aligned}
$$

Proof. Just as above, using Lemmas 2.1, 2.2, and taking into account that, according to (4.3), $\int \omega_{0 u}(\eta) \Omega^{\prime}(\eta) \widetilde{\Omega}^{\prime \prime}(\eta) d \eta=0, \int \omega_{0 u}(\eta) \widetilde{\Omega}^{\prime \prime}(\eta) d \eta=0, \int \Omega^{\prime}(\eta) \widetilde{\Omega}^{\prime \prime}(\eta) d \eta=0$, one can calculate

$$
\begin{gathered}
u(x, t, \varepsilon) v(x, t, \varepsilon)=u_{0} v_{0}+[u v] H(-x+\phi(t)) \\
+e(t)\left(u_{0}+u_{1} \int \omega_{0 u}(\eta) \omega_{\delta}(\eta) d \eta\right. \\
\left.+Q(t) \int \omega_{\delta}(\eta) \Omega^{\prime}(\eta) d \eta\right) \delta(-x+\phi(t))+O_{\mathcal{D}^{\prime}}(\varepsilon), \\
u^{2}(x, t, \varepsilon) v(x, t, \varepsilon)=u_{0}^{2} v_{0}+\left[u^{2} v\right] H(-x+\phi(t)) \\
+\left\{e ( t ) \left(u_{0}^{2}+2 u_{0} u_{1} \int \omega_{0 u}(\eta) \omega_{\delta}(\eta) d \eta+u_{1}^{2} \int \omega_{0 u}^{2}(\eta) \omega_{\delta}(\eta) d \eta\right.\right. \\
+Q^{2}(t) \int\left(\Omega^{\prime}\right)^{2}(\eta) \omega_{\delta}(\eta) d \eta+2 Q(t)\left(u_{0} \int \Omega^{\prime}(\eta) \omega_{\delta}(\eta) d \eta\right. \\
\left.\left.+u_{1} \int \omega_{0 u}(\eta) \Omega^{\prime}(\eta) \omega_{\delta}(\eta) d \eta\right)\right) \\
\left.+R(t) u_{1}^{2} \int \omega_{0 u}^{2}(\eta) \widetilde{\Omega}^{\prime \prime}(\eta) d \eta\right\} \delta(-x+\phi(t))+O_{\mathcal{D}^{\prime}}(\varepsilon) .
\end{gathered}
$$

Substituting into the first equation of system (1.9) smooth ansatzs (1.20), asymptotics (4.5), (4.6), and setting the left-hand side equal to zero, we obtain the necessary and sufficient conditions for the equality $L_{31}[u(x, t, \varepsilon), v(x, t, \varepsilon)]=O_{\mathcal{D}^{\prime}}(\varepsilon)$ :

$$
\begin{aligned}
L_{31}\left[u_{+}, v_{+}\right] & =0, \quad x>\phi(t), \\
L_{31}\left[u_{-}, v_{-}\right] & =0, \quad x<\phi(t), \\
\dot{e}(t) & =\left.([u v]-[v] \dot{\phi}(t))\right|_{x=\phi(t)}, \\
\dot{\phi}(t) & =\left.\left(u_{0}+u_{1} a_{1}+Q(t) b_{1}\right)\right|_{x=\phi(t)}
\end{aligned}
$$

Substitute smooth ansatzs (1.20), and asymptotics (4.5), (4.6) into the second equation of system (1.9). Next, taking into account the third relation of (4.7), we obtain the 
necessary and sufficient conditions for the equality $L_{32}[u(x, t, \varepsilon), v(x, t, \varepsilon)]=O_{\mathcal{D}^{\prime}}(\varepsilon)$ :

$$
\begin{aligned}
L_{32}\left[u_{+}, v_{+}\right]= & 0, \quad x>\phi(t), \\
L_{32}\left[u_{-}, v_{-}\right]= & 0, \quad x<\phi(t), \\
\frac{d(e(t) \dot{\phi}(t))}{d t}= & \left.\left(\left[u^{2} v\right]-[u v] \dot{\phi}(t)\right)\right|_{x=\phi(t)}, \\
e(t)(\dot{\phi}(t))^{2}= & \left\{e ( t ) \left(u_{0}^{2}+2 u_{0} u_{1} a_{1}+u_{1}^{2} a_{2}\right.\right. \\
& \left.+Q^{2}(t) b_{2}+2 Q(t)\left(u_{0} b_{1}+u_{1} c_{1}\right)\right) \\
& \left.+R(t) u_{1}^{2} c_{2}\right\}\left.\right|_{x=\phi(t)}
\end{aligned}
$$

From (4.7), (4.8) we have system (4.4).

It remains to point out that, repeating the second stage of the proof of Theorems 2.3 and 3.1 almost word for word, we obtain that there exists $T>0$ such that system (4.4) is solvable.

We note that the obtained system of equations on the trajectory $x=\phi(t)$ (the fifth and sixth equations in system (4.4)), i.e., the Rankine-Hugoniot conditions, have the second order. Hence, for (4.4) to be uniformly solvable, in addition to the (natural) initial values $e(0)$ and $\phi(0)$, it is necessary to specify an additional initial value $\dot{\phi}(0)$.

Clearly, if the initial values $\left.u_{ \pm}\right|_{t=0}$ and $\dot{\phi}(0)$ are matched in the sense that inequality (4.1) holds for $t=0$, then there exists $T>0$ such that, for $0 \leq t \leq T$, inequality (4.1) holds. Hence the values $u_{ \pm}$and $v_{ \pm}$on the curve $x=\phi(t), 0 \leq t \leq T$, are determined. This implies that the Rankine-Hugoniot conditions (the fifth and sixth equations in system (4.4)) are well defined and solvable for $0 \leq t \leq T$.

4.2. Construction of a generalized solution. We obtain a generalized solution of the Cauchy problem as a weak limit of a weak asymptotic solution constructed in Theorem 4.1 .

Theorem 4.2. Let inequality (4.1) hold for $t=0$, then there exists $T>0$ such that the Cauchy problem (1.9), (1.14) for $t \in[0, T)$ has a unique generalized solution

$$
\begin{aligned}
& u(x, t)=u_{+}(x, t)+[u(x, t)] H(-x+\phi(t)), \\
& v(x, t)=v_{+}(x, t)+[v(x, t)] H(-x+\phi(t))+e(t) \delta(-x+\phi(t)),
\end{aligned}
$$

which satisfies the integral identities (1.16). Here $V(x, t)=v_{0}(x, t)+v_{1}(x, t) H(-x+\phi(t))$, and functions $u_{ \pm}(x, t), v_{ \pm}(x, t), \phi(t), e(t)$ are defined by the system

$$
\begin{aligned}
L_{31}\left[u_{+}, v_{+}\right] & =0, \quad x>\phi(t), \\
L_{31}\left[u_{-}, v_{-}\right] & =0, \quad x<\phi(t), \\
L_{32}\left[u_{+}, v_{+}\right] & =0, \quad x>\phi(t), \\
L_{32}\left[u_{-}, v_{-}\right] & =0, \quad x<\phi(t), \\
\dot{e}(t) & =\left.([u v]-[v] \dot{\phi}(t))\right|_{x=\phi(t)}, \\
\frac{d(e(t) \dot{\phi}(t))}{d t} & =\left.\left(\left[u^{2} v\right]-[u v] \dot{\phi}(t)\right)\right|_{x=\phi(t)},
\end{aligned}
$$

where initial data are defined from (1.14). 
Proof. Substituting the correction functions $Q(t), R(t)$ given by the two last equations of system (4.4), into (4.5), (4.6), we obtain the following relations:

$$
\begin{gathered}
u(x, t, \varepsilon) v(x, t, \varepsilon)=u_{0}(x, t) v_{0}(x, t)+[u(x, t) v(x, t)] H(-x+\phi(t)) \\
+e(t) \dot{\phi}(t) \delta(-x+\phi(t))+O_{\mathcal{D}^{\prime}}(\varepsilon), \\
u^{2}(x, t, \varepsilon) v(x, t, \varepsilon)=u_{0}^{2}(x, t) v_{0}(x, t)+\left[u^{2}(x, t) v(x, t)\right] H(-x+\phi(t)) \\
+e(t)(\dot{\phi}(t))^{2} \delta(-x+\phi(t))+O_{\mathcal{D}^{\prime}}(\varepsilon), \quad \varepsilon \rightarrow+0 .
\end{gathered}
$$

According to Theorem 4.1 we have the following relations:

$$
L_{31}[u(x, t, \varepsilon), v(x, t, \varepsilon)]=O_{\mathcal{D}^{\prime}}(\varepsilon), \quad L_{32}[u(x, t, \varepsilon), v(x, t, \varepsilon)]=O_{\mathcal{D}^{\prime}}(\varepsilon) .
$$

The proof of the first integral identity (1.16) is based on relation (4.10) and the same calculations as those carried out above in Subsec. 2.2. and we omit them here.

According to Theorem 4.1, $L_{32}[u(x, t, \varepsilon), v(x, t, \varepsilon)]=O_{\mathcal{D}^{\prime}}(\varepsilon)$. Let us apply the lefthand and right-hand sides of this relation to an arbitrary test function $\varphi(x, t) \in \mathcal{D}(\mathbb{R} \times$ $[0, T)$ ). Then integrating by parts, we obtain

$$
\begin{gathered}
\int_{0}^{T} \int\left(u(x, t, \varepsilon) v(x, t, \varepsilon) \varphi_{t}(x, t)+u^{2}(x, t, \varepsilon) v(x, t, \varepsilon) \varphi_{x}(x, t)\right) d x d t \\
+\int u(x, 0, \varepsilon) v(x, 0, \varepsilon) \varphi(x, 0) d x=O(\varepsilon) .
\end{gathered}
$$

Passing to the limit as $\varepsilon \rightarrow+0$ and taking into account (4.10), (4.11), and (2.10), (2.11), we obtain integral identities (2.7):

$$
\begin{gathered}
\int_{0}^{T} \int\left(u V \varphi_{t}+u^{2} V \varphi_{x}\right) d x d t+\int u^{0}(x) V^{0}(x) \varphi(x, 0) d x \\
+\int_{0}^{T} e(t) \dot{\phi}(t)\left(\varphi_{t}(\phi(t), t)+\dot{\phi}(t) \varphi_{x}(\phi(t), t)\right) d t+e^{0} \dot{\phi}(0) \varphi(0,0)=0 .
\end{gathered}
$$

Here $\Gamma=\{(x, t): x=\phi(t), t \in[0, T)\}$, and

$$
\int_{\Gamma} e(x, t) \dot{\phi}(t) \frac{\partial \varphi}{\partial \mathbf{l}} d l=\int_{0}^{T} e(t) \dot{\phi}(t)\left(\varphi_{t}(\phi(t), t)+\dot{\phi}(t) \varphi_{x}(\phi(t), t)\right) d t .
$$

If the initial data (1.14) are piecewise constant, i.e., $u_{+}=u_{0}^{0}, u_{-}=u_{0}^{0}+u_{1}^{0}, v_{+}=v_{0}^{0}$, $v_{-}=v_{0}^{0}+v_{1}^{0}, \quad u_{1}^{0}=[u]>0$, then we have the following statement from Theorem 4.2 ,

Theorem 4.3. The Cauchy problem (1.9) with piecewise constant initial data (1.14) for $t \in[0, \infty)$ has a unique generalized solution

$$
\begin{aligned}
& u(x, t)=u_{+}+[u] H(-x+\phi(t)), \\
& v(x, t)=v_{+}+[v] H(-x+\phi(t))+e(t) \delta(-x+\phi(t)),
\end{aligned}
$$

which satisfies the integral identities (1.16), where 
(i) if $[v] \neq 0$, then

$$
\begin{aligned}
e(t) & =\sqrt{\left(e^{0}\right)^{2}+2 e^{0} \dot{e}(0) t+\left([u v]^{2}-[v]\left[u^{2} v\right]\right) t^{2}} \\
\phi(t) & =\frac{e^{0}+[u v] t-\sqrt{\left(e^{0}\right)^{2}+2 e^{0} \dot{e}(0) t+\left([u v]^{2}-[v]\left[u^{2} v\right]\right) t^{2}}}{[v]},
\end{aligned}
$$

and $\dot{e}(0)=[u v]-[v] \dot{\phi}(0)$,

(ii) if $[v]=0$, then

$$
\begin{aligned}
e(t) & =e^{0}+[u] v_{0} t, \\
\phi(t) & =\frac{e^{0} \dot{\phi}(0)+\frac{1}{2} v_{0}\left[u^{2}\right] t}{e^{0}+v_{0}[u] t} t .
\end{aligned}
$$

Proof. In this case from Theorem 4.2 we have the Rankine-Hugoniot conditions:

$$
\begin{aligned}
\dot{e}(t) & =[u v]-[v] \dot{\phi}(t), \\
\frac{d(e(t) \dot{\phi}(t))}{d t} & =\left[u^{2} v\right]-[u v] \dot{\phi}(t) .
\end{aligned}
$$

Let $[v] \neq 0$. Substituting $\dot{\phi}(t)$ from the first equation into the second one, we obtain

$$
e \ddot{e}(t)+(\dot{e}(t))^{2}=[u v]^{2}-[v]\left[u^{2} v\right] .
$$

Integrating the last expression, we obtain

$$
e(t) \dot{e}(t)=\frac{1}{2} \frac{d\left(e^{2}(t)\right)}{d t}=A_{1}+A t,
$$

where $A_{1}$ is a constant,

$$
\begin{aligned}
A=[u v]^{2}-[v]\left[u^{2} v\right] & \\
=\left(u_{-} v_{-}-u_{+} v_{+}\right)^{2}-\left(v_{-}\right. & \left.-v_{+}\right)\left(u_{-}^{2} v_{-}-u_{+}^{2} v_{+}\right) \\
& =v_{-} v_{+}\left(u_{-}-u_{+}\right)^{2} \geq 0 .
\end{aligned}
$$

Thus we have

$$
e(t)=\sqrt{A_{0}+2 A_{1} t+A t^{2}}, \quad A_{0}=\left(e^{0}\right)^{2} .
$$

Hence

$$
\dot{e}(t)=\frac{A_{1}+A t}{\sqrt{\left(e^{0}\right)^{2}+2 A_{1} t+A t^{2}}}, \quad A_{1}=e^{0} \dot{e}(0),
$$

and

$$
\begin{aligned}
\dot{\phi}(t) & =\frac{[u v]-\dot{e}(t)}{[v]}, \\
\phi(t) & =\frac{e^{0}+[u v] t-\sqrt{\left(e^{0}\right)^{2}+2 e^{0} \dot{e}(0) t+A t^{2}}}{[v]} .
\end{aligned}
$$

If $[v]=0$, then $v_{-}=v_{+}=v_{0}$. In this case $e(t)=e^{0}+[u] v_{0} t$. Substituting the last relation into the second equation of system (4.12), we have

$$
e(t) \ddot{\phi}(t)+2[u] v_{0} \dot{\phi}(t)=\left[u^{2}\right] v_{0} .
$$

Integrating this differential equation, after elementary calculations, we obtain

$$
\dot{\phi}(t)=\frac{\left[u^{2}\right]}{2[u]}+\left(\dot{\phi}(0)-\frac{\left[u^{2}\right]}{2[u]}\right) \frac{\left(e^{0}\right)^{2}}{\left(e^{0}+t v_{0}[u]\right)^{2}} .
$$


Hence $\phi(t)=\frac{e^{0} \dot{\phi}(0)+t v_{0}\left[u^{2}\right] / 2}{e^{0}+t v_{0}[u]} t$.

Now we prove that the constructed solution is an entropy solution. Consider the case $[v] \neq 0$. Since $u_{+} \leq \dot{\phi}(0) \leq u_{-}$, it is easy to show that $\dot{e}(0)>0$. Hence $t=-\frac{A_{1}}{A}=$ $\frac{e^{0} \dot{e}(0)}{v_{-} v_{+}[u]^{2}}<0$, i.e., $\left(e^{0}\right)^{2}+2 e^{0} \dot{e}(0) t+A t^{2}>0$ for all $t \geq 0$. From (4.14), (4.15) we obtain

$$
\ddot{\phi}(t)=\left(e^{0}\right)^{2} \frac{(\dot{e}(0))^{2}-v_{-} v_{+}[u]^{2}}{[v]\left(\left(e^{0}\right)^{2}+2 e^{0} \dot{e}(0) t+v_{-} v_{+}[u]^{2} t^{2}\right)^{3 / 2}} .
$$

Thus $\dot{\phi}(t)$ and $\dot{e}(t)$ are monotonous functions for all $t \geq 0$.

Consequently, taking into account that

$$
\begin{aligned}
\dot{e}(t) & \rightarrow \sqrt{A}=[u] \sqrt{v_{-} v_{+}} \\
\dot{\phi}(t) & \rightarrow \frac{u_{-} \sqrt{v_{-}}+u_{+} \sqrt{v_{+}}}{\sqrt{v_{-}}+\sqrt{v_{+}}}, \quad t \rightarrow \infty,
\end{aligned}
$$

and relations

$$
\begin{array}{lcl}
u_{+} \leq & \dot{\phi}(0) & \leq u_{-}, \\
u_{+} \leq & \frac{u_{-} \sqrt{v_{-}}+u_{+} \sqrt{v_{+}}}{\sqrt{v_{-}}+\sqrt{v_{+}}} & \leq u_{-},
\end{array}
$$

one can easily see that the entropy condition (4.1) holds.

The case $[v]=0$ can be considered in the same way.

Corollary 4.4. Let $\dot{\phi}(0)=\frac{u_{-} \sqrt{v_{-}}+u_{+} \sqrt{v_{+}}}{\sqrt{v_{-}}+\sqrt{v_{+}}}$, i.e., $\dot{e}(0)=[u] \sqrt{v_{-} v_{+}}$. Then the Cauchy problem (1.9) with piecewise constant initial data (1.14) for $t \in[0, \infty)$ has a unique generalized solution

$$
\begin{aligned}
& u(x, t)=u_{+}+[u] H(-x+\phi(t)), \\
& v(x, t)=v_{+}+[v] H(-x+\phi(t))+e(t) \delta(-x+\phi(t)),
\end{aligned}
$$

satisfies the integral identities (1.16), where

$$
\begin{aligned}
\phi(t) & =\frac{u_{-} \sqrt{v_{-}}+u_{+} \sqrt{v_{+}}}{\sqrt{v_{-}}+\sqrt{v_{+}}} t, \\
e(t) & =e^{0}+\sqrt{v_{-} v_{+}}\left(u_{-}-u_{+}\right) t .
\end{aligned}
$$

Note that, according to Theorem 4.3 and Corollary 4.4, if

$$
\dot{e}(0)=[u] \sqrt{v_{-} v_{+}},
$$

the trajectory of $\delta$-shock wave $x=\phi(t)$ has the same form as for $e(0)=0$.

\section{Acknowledgements}

The authors are greatly indebted to V. I. Polischook and Y. Belopolskaya for fruitful discussions.

\section{REFERENCES}

[1] F. Bouchut, On zero pressure gas dynamics, in "Advances in Kinetic Theory and Computing", Series on Advances in Mathematics for Applied Sciences, 22, 171-190, World Scientific, River Edge, NJ, 1994. MR1323183 (96e:76107)

[2] V. G. Danilov, V. P. Maslov, V. M. Shelkovich, Algebra of singularities of singular solutions of first-order quasilinear strictly hyperbolic systems. Theor. Math. Phys. 114 (1998), no. 1, 1-42. MR 1756560 (2001f:35244) 
[3] V. G. Danilov, G. A. Omel'yanov, V. M. Shelkovich, Weak Asymptotics Method and Interaction of Nonlinear Waves, in Mikhail Karasev (ed.), "Asymptotic Methods for Wave and Quantum Problems", Amer. Math. Soc. Transl., Ser. 2, 208, 2003, 33-163. MR1995392 (2004f:35021)

[4] V. G. Danilov, V. M. Shelkovich, Propagation and interaction of nonlinear waves to quasilinear equations, Hyperbolic problems: Theory, Numerics, Applications (Eighth International Conference in Magdeburg, February/March 2000, v.I). International Series of Numerical Mathematics, v. 140, Birkhäuser, Basel/Switzerland, 2001, 267-276. MR.1882927

[5] V. G. Danilov and V. M. Shelkovich, Propagation and interaction of shock waves of quasilinear equation. Nonlinear Studies 8 (2001), no. 1, 135-169. MR.1856223 (2002k:35199)

[6] V. G. Danilov, V. M. Shelkovich, Propagation and interaction of delta-shock waves of a hyperbolic system of conservation laws, In Hou, Thomas Y.; Tadmor, Eitan (Eds.), Hyperbolic Problems: Theory, Numerics, Applications. Proceedings of the Ninth International Conference on Hyperbolic Problems held in CalTech, Pasadena, March 25-29, 2002, Springer-Verlag, 2003, 483-492. MR2053197 (2005a:35191)

[7] V. G. Danilov, V. M. Shelkovich, Dynamics of propagation and interaction of $\delta$-shock waves in hyperbolic systems. pp.40, Preprint 2003-068 at the url:

http://www.math.ntnu.no/conservation/2003/068.html (to appear in the Journal of Differential Equations)

[8] V. G. Danilov, V. M. Shelkovich, Propagation and interaction of $\delta$-shock waves to hyperbolic systems of conservation laws. Dokl. Akad. Nauk 394 (2004), no. 1, 10-14. English transl. in Russian Doklady Mathematics 69 (2004), no. 1. MR2088475

[9] Weinan E., Yu. Rykov, Ya. G. Sinai, Generalized variational principles, global weak solutions and behavior with random initial data for systems of conservation laws arising in adhesion particle dynamics. Comm. Math. Phys. 177 (1996), 349-380. MR.1384139 (98a:82077)

[10] G. Ercole, Delta-shock waves as self-similar viscosity limits. Quart. Appl. Math. 58 (2000), no. 1, 177-199. MR1739044 (2000j:35187)

[11] Jiaxin $\mathrm{Hu}$, The Riemann problem for pressureless fluid dynamics with distribution solutions in Colombeau's sense. Comm. Math. Phys. 194 (1998), 191-205. MR1628318 (99e:35130)

[12] Feiming Huang, Existence and uniqueness of discontinuous solutions for a class of nonstrictly hyperbolic systems, In Chen, Gui-Qiang (ed.) et al. Advances in nonlinear partial differential equations and related areas. Proceeding of conf. dedicated to Prof. Xiaqi Ding, China, 1997, 187-208. MR.1690829 (2000c:35147)

[13] K. T. Joseph, A Riemann problem whose viscosity solutions contain $\delta$-measures. Asymptotic Analysis 7 (1993), 105-120. MR 1225441 (94f:35083)

[14] B. Lee Keyfitz and H. C. Kranzer, Spaces of weighted measures for conservation laws with singular shock solutions. Journal of Differential Equations 118 (1995), 420-451. MR.1330835 (96b:35138)

[15] P. Le Floch, An existence and uniqueness result for two nonstrictly hyperbolic systems,25-FEB-2005 Nonlinear Evolution Equations That Change Type, Springer-Verlag, 1990, 126-138. MR1074190 (91g:35171)

[16] J. Li and Tong Zhang, On the initial-value problem for zero-pressure gas dynamics, Hyperbolic problems: Theory, Numerics, Applications. Seventh International Conference in Zürich, February 1998, Birkhäuser, Basel, Boston, Berlin, 1999, 629-640. MR1717235(2000f:76114)

[17] Tai-Ping Liu, Zhouping Xin, Overcompressive shock waves, Nonlinear evolution equations that change type, Springer-Verlag, 1990, 139-145. MR1074191 (91f:35171)

[18] A. Majda, Compressible fluid flow and systems of conservation laws in several space variables, Springer-Verlag, New York, 1984. MR0748308 (85e:35077)

[19] G. Dal Maso, P. G. Le Floch, and F. Murat, Definition and weak stability of nonconservative products. J. Math. Pures Appl. 74 (1995), 483-548. MR.1365258 (97b:46052)

[20] M. Nedeljkov, Delta and singular delta locus for one-dimensional systems of conservation laws. Math. Meth. Appl. Sci. 27 (2004) 931-955. MR2055283

[21] B. L. Rozhdestvenskii and N. N. Yanenko, Systems of Quasilinear Equations, Moscow, Nauka, 1978 (in Russian) B. L. Rozhdestvenskii and N. N. Janenko, Systems of Quasilinear Equations and Their Applications to Gas Dynamics, New York, Am. Math., 1983. MR0694243 (85f:35127)

[22] S. F. Shandarin and Ya. B. Zeldovich, The large-scale structure of the universe: turbulence, intermittency, structures in a self-gravitating medium. Rev. Mod. Phys. 61 (1989), 185-220. MR0989562 (89m:85008) 
[23] V. M. Shelkovich, An associative-commutative algebra of distributions that includes multipliers, generalized solutions of nonlinear equations. Mathematical Notices 57 (1995), no. 5, 765-783. MR 1347378 (96g:46025)

[24] V. M. Shelkovich, Delta-shock waves of a class of hyperbolic systems of conservation laws, in A. Abramian, S. Vakulenko, V. Volpert (Eds.), "Patterns and Waves", AkademPrint, St. Petersburg, 2003, 155-168. MR2014201 (2004i:35220)

[25] Wancheng Sheng, Tong Zhang, The Riemann problem for the transportation equations in gas dynamics. Memoirs of the Amer. Math. Soc., 137, no. 654, (1999), 1-77. MR1466909 (99g:35109)

[26] De Chun Tan, Tong Zhang and Yu Xi Zheng, Delta-shock waves as limits of vanishing viscosity for hyperbolic systems of conservation laws. Journal of Differential Equations 112 (1994), 1-32. MR $1287550(95 \mathrm{~g}: 35124)$

[27] A. I. Volpert, The space BV and quasilinear equations. Math. USSR Sb. 2 (1967), 225-267.

[28] Hanchun Yang, Riemann problems for a class of coupled hyperbolic systems of conservation laws. Journal of Differential Equations 159 (1999), 447-484. MR1730728 (2000j:35184)

[29] Ya. B. Zeldovich, Gravitationnal instability: An approximate theory for large density perturbations. Astron. Astrophys. 5 (1970), 84-89. 